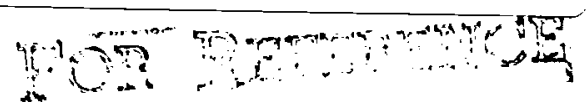

\title{
The Structure of the Vorticity Field in Turbulent Channel Flow. Part II: Study of Ensemble-Average Fields
}

John Kim and Parviz Moin

October 1984

LERMAR Gory

16:? 1984

LANGLEY RESEARSH CENTER

LIERARY, NASA

HAPTON, VIRGINIA

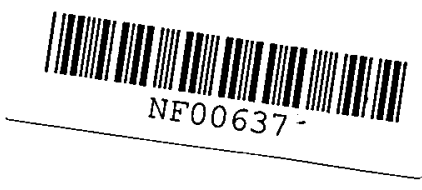




\section{The Structure of the Vorticity Field in Turbulent Channel Flow. Part II: Study of Ensemble-Average Fields}

John Kim,

Parviz Moin, Ames Research Center, Moffett Field, California 


\section{The structure of the vorticity field in turbulent channel flow.}

\section{Part 2. Study of ensemble-averaged fields}

JOHN KIM AND PARVIZ MOIN

NASA Ames Research Center, Moffett Field, California 94035

Several conditional sampling techniques are applied to a data base generated by large-eddy simulation of turbulent channel flow. It is shown that the bursting process is associated with well-organized horseshoe vortices inclined at about $45^{\circ}$ to the wall. These vortical structures are identified by examining the vortex lines of three-dimensional, ensemble-averaged vorticity fields. Two distinct horseshoeshaped vortices corresponding to the sweep and ejection events are detected. These vortices are associated with high Reynolds shear stress and hence make a significant contribution to turbulent energy production.

\section{Introduction}

In Part 1 (Moin \& Kim 1984), an investigation of the existence of horseshoe (or hairpin) vortex structures in turbulent channel flow was reported in which a data base generated by the large-eddy simulation technique was used. It was shown that in a large portion of the flow, the distribution of the inclination angle of vorticity vectors attains its maximum at about $45^{\circ}$ to the wall. Two-point correlations of velocity and vorticity fluctuations along lines inclined at $45^{\circ}$ and $135^{\circ}$ to the flow direction provided strong evidence for the existence of vortical structures inclined at $45^{\circ}$. to the wall. In addition to these statistical analyses, the vorticity vectors and vortex lines of instantaneous three-dimensional vorticity fields also indicated the presence of horseshoe vortical structures. It was shown that these horseshoeshaped vortex lines are a result of the deformation of the primary vortex lines, which point to the spanwise directions when undisturbed. 
In the present paper, we further study the structure of the vorticity field in turbulent channel flow, using various conditional sampling techniques. The objective of this work is to identify the vortical structures associated with the bursting process in the wall-bounded turbulent flows. The bursting process is composed of a sequence of quasi-cyclic events that occur in the wall region of turbulent flows; it is responsible for about $70 \%$ of total turbulence production (see Kim, Kline, and Reynolds 1971; Kline 1978), Our aim is to determine if the organized structures associated with the bursting process are indeed horseshoe vortices, as was first hypothesized by Kline, Reynolds, Schraub \& Runstadler (1967). The dependency of the ensemble-averaged vortical structures on the detection criteria, and the question of whether this ensemble-averaged structure is an artifact of the ensemble-averaging process are examined. The ensemble-averaged pattern of these vortical structures that emerge from the analysis could provide the basis for a hypothetical model of the organized structures of wall-bounded shear flows.

The same data base used in Part 1 is used here. That data base was obtained by large-eddy simulation of a fully developed turbulent channel flow (Moin \& Kim 1982) for a Reynolds number of 13800 , based on the centerline velocity and channel half-width. Three-dimensional velocity and pressure fields were stored at widely separated flow times. This data base has also been used in previous investigations of the organized turbulence structures (Kim 1983; Moin 1984) and has provided complementary information toward our current understanding of the coherent motions in turbulent flows.

In $\S 2, \mathrm{a}$ brief description of several conditional sampling techniques is presented. The results obtained by applying those techniques to the aforementioned data base are given in $\S 3$. Discussion of these results is presented in $\S 4$, and a summary is provided in $\S 5$. 


\section{Conditional sampling techniques}

Previous investigations of the structures of wall-layer turbulence (Kim 1983, 1984) using a variant of the VITA sampling technique of Blackwelder \& Kaplan (1976) indicated that the bursting process is associated with a pair of counterrotating streamwise vortices. Whether these streamwise vortices are indeed the "legs" of a horseshoe vortex structure could not be established, because only the streamwise component of the ensemble-averaged vorticity field was examined in two-dimensional planes. To address this question, the VISA (variable-interval space-averaging) sampling technique of $\operatorname{Kim}(1983,1984)$, in addition to other conditional sampling techniques, is used in the present work. The VISA technique is essentially the same as the VITA technique, except that VISA produces a spatial structure instead of a temporal structure. The exact definition of this conditional sampling technique is given in table 1, along with the other techniques used in this work. However, as is discussed in more detail in the next section, sampling in the VISA technique is not triggered by the passage of horseshoe vortices. This is because the sampling in the VITA technique (especially the later version of Chen \& Blackwelder 1978) is basically triggered by a sweeping event, that is, a region of high-speed fluid moving toward the wall causing a local acceleration near the wall (Corino \& Brodkey 1969). In order to have a detection scheme triggered by the ejection of low-speed fluid from the wall region (ejection event), which is more likely to be associated with horseshoe vortical structures, the location of the detection point was moved away from the wall to $y^{+}=100,200$, and $300 .^{1}$ In addition, the detection function was set such that it only detects a local deceleration, ${ }^{2}$ which is normally associated with

\footnotetext{
${ }^{1}$ The superscript + indicates a nondimensional quantity scaled by the wall variables; e.g., $y^{+}=$ $y u_{r} / \nu$, where $\nu$ is the kinematic viscosity and $u_{r}=\sqrt{\tau_{w} / \rho}$ is the wall shear velocity.

${ }^{2}$ Note that the terms acceleration and deceleration are used to describe the velocity measured by a fixed probe in space. Corresponding velocity distribution in the streamwise direction would be a negative gradient of the streamwise velocity for the acceleration and a positive gradient for the deceleration.
} 
the ejection event. These techniques are referred to as VISA-1, 2, and 3 in table 1. Anticipating that the induced velocities of horseshoe vortices would produce high Reynolds shear stress, the quadrant analysis (Willmarth \& Lu 1972; Wallace, Eckelmann \& Brodkey 1972; Alfredsson \& Johansson 1984) is also applied to the same data base. Of particular interest are the second $\left(u^{\prime}<0, v^{\prime}>0\right)$ and the fourth $\left(u^{\prime}>0, v^{\prime}<0\right)$ quadrants, which make positive contributions to turbulent energy production. With this technique, vortical structures associated with the sweep and the ejection events can be separated. These techniques will be referred to as QD-2 and QD-4 in the remainder of this paper. In addition, in an attempt to measure the contribution of the detected vortical structures to the Reynolds shear stress, different sizes of threshold intervals are added to the quadrant analysis instead of using the "hole" technique of Lu \& Willmarth (1973), which discriminates events associated with the Reynolds stress smaller than a threshold value. These techniques are referred to as QD-A and QD-B in table 1.

Ensemble-averaged structures obtained using these conditional sampling techniques will depend on the detection criteria of each technique. The educed structures, therefore, are the structures associated with the particular events that the conditional sampling techniques are designed to detect: for example, structures detected by QD-2 are associated with the ejection event, whereas structures detected by QD-4 are associated with the sweep event. Many different structures occur in turbulent flows, and a special technique can be devised to detect a particular structure. Important structures are those that play a significant role in the dynamics of the flow.

All the results presented in the following section are obtained from ensemble averages of more than 1000 events. Some of the results (VISA) are averaged over more than 3000 events. The increased sample size removes statistical jitter without affecting the characteristics of the educed structures. The coordinate system used in this paper is shown in figure 1 . Here, $x, y$, and $z$ represent the streamwise, normal 
(to the wall), and spanwise directions, respectively; $u, v$, and $w$ denote the velocity components along $x, y$, and $z$, respectively. The origin of the streamwise coordinate, $x=0$, and the spanwise coordinate, $z=0$, corresponds to the location of the detection point, and $y$ is measured from the wall. The length scales are nondimensionalized by the wall parameters, and, for the Reynolds number considered here, the channel half width corresponds to $\delta^{+} \approx 640$.

\section{Ensemble-averaged vortical structures}

As indicated in Part 1 (Moin \& Kim 1984) the most direct method of identifying vortical structures, and in particular the horseshoe vortices, is tracing vortex lines in three-dimensional space. The location of a vortex line in a three-dimensional space, $\mathbf{x}$, is defined by

$$
\frac{d x}{d s}=\frac{\omega}{|\omega|},
$$

where $s$ is the distance along the vortex line and $\omega$ is the vorticity vector. In Part 1, instantaneous vortex lines in turbulent channel flow illustrated the threedimensional structure of the horseshoe vortices. In this section, vortex lines of the ensemble-average vorticity fields ${ }^{1}$ are presented. They are obtained from the various conditional sampling techniques described in $\S 2$ and represent the ensembleaveraged shape of the vortical structures.

In figure 2, several randomly chosen vortex lines in an instantaneous computed vorticity field are shown. The chaotic nature of the flow is quite apparent but several horseshoe-shaped vortex lines can be identified. As will be shown below, some of the conditional sampling techniques detect such a structure and the resulting ensemble-averaged patterns display a pronounced horseshoe (or hairpin) shape. In

\footnotetext{
${ }^{1}$ Note that for all the conditional sampling techniques used in the present work, the vorticity of an ensemble-averaged velocity field is the same as the ensemble-averaged vorticity field. See the definitions of the ensemble-averages in table 1.
} 
some techniques other structures, as well as the horseshoe vortices, trigger sampling, and the ensemble-averaged patterns show a similar but a weaker structure.

The vortex lines of the ensemble-averaged vorticity field obtained using VISA are shown in figure 3. Only the vortex lines in the neighborhood of the detection point $(x=0, z=0)$ are shown in the figure; the vortex lines that approach to $y^{+} \approx 55$ as $z \rightarrow \infty$ for several $x$-locations upstream and downstream of the detection point are shown in figure $3 a$; those that approach $y^{+} \approx 106$ for the same streamwise locations are shown in figure $3 b$. Figure $3 a$ shows the existence of very weak horseshoe-type vortical structures downstream of the detection point $(x>0)$. The ensembleaveraged field contains appreciable streamwise and normal vorticity components (their time-averaged values are zero), but their magnitudes are relatively small compared with that of the spanwise component. Upstream of the detection point, a different vortical structure is noticeable; it becomes more pronounced at higher $y^{+}$ locations (figure $3 b$ ). The apexes of these vortex lines are located upstream of their legs and closer to the wall. The induced fluid motion from this "inverted" horseshoe vortical structure corresponds to the sweep event, whereas an induced fluid motion owing to the "regular" horseshoe vortices corresponds to the ejection of fluid away from the wall. Note that the inverted horseshoe is located upstream of the regular horseshoe and that the detection point is in the middle of these two structures. For a probe fixed in space, the passage of these structures would first register positive normal velocity followed by a negative one in time; this is in agreement with the experimental measurements using the VITA technique (Blackwelder \& Kaplan 1976; Alfredsson \& Johansson 1984).

It should be pointed out that the boundaries of the ensemble-averaged structures are smeared out, since the instantaneous structures appear randomly in time and space with jitter in their size, shape, and strength (induced velocity). Therefore, these ensemble-averaged structures are not confined to a compact region in space 
and should not be considered as representative of instantaneous structures. However, the shape of the vortex lines composing them are indicative of the general shapes of the detected structure.

The perturbation ensemble-averaged vorticity field is obtained by subtracting the mean component of vorticity from the ensemble-averaged field. The vortex lines corresponding to this perturbation field are shown in figure 4 . The lines shown are those that pass through the point of the maximum perturbation vorticity for a given streamwise location; that is, the starting point for the integration of equation (1) was the point of maximum perturbation vorticity in the $(y, z)$-plane for a given $x$. These vortex lines display ring-like loops. Note the direction of the vortex loops indicated by arrows. Two distinct groups of loops exist upstream and downstream of the detection point corresponding to the sweep and the ejection motions. The ensemble-averaged vorticity field can be represented by straight vortex lines in the spanwise direction (mean vorticity) plus the ring vortices (perturbation). This is similar to the initial condition used by Leonard (1980) for the numerical simulation of a turbulent spot, in which ring vortices were superposed on to the mean vorticity.

Figure 5 displays the vortex lines drawn from the ensemble-averaged field obtained using VISA-1. Recall that VISA-1 is modified to detect the flow deceleration which is normally associated with ejection of low-speed fluid away from the wall. Again, only the vortex lines near the detection point are shown. The vortex lines in figures $5(a-d)$ approach $y^{+} \approx 20,55,143$, and 300 , respectively, as $z \rightarrow \infty$; these figures display the difference in the vortical structures as a function of the distance from the wall. Close to the wall (figure $5 a$ ), all the vortex lines are parallel to the spanwise direction (direction of mean vorticity) indicating no apparent organized structure there; a pronounced horseshoe structure near the detection point is shown in figure $5 b$; the two vortical structures associated with the sweep and ejection are shown in figure $5 c$; and in figure $5 d$, no vortical structure is noticeable far away from the wall. In figure $5 c$, the "inverted" structures are located upstream and above the "regular" 
horseshoe vortices. When the detection point is moved farther away from the wall $\left(y^{+}=200\right.$, VISA-2), both the regular and inverted horseshoe structures become more pronounced, as shown in figure 6 . The $y$-locations in figures $5 b$ and $5 c$, and $6 a$ and $6 b$ correspond to the locations of the most pronounced horseshoe structures for each case. To illustrate variations of the vortical structure in the $y$-direction, vortex lines approaching various $y$-locations as $z \rightarrow \infty$ for a given streamwise location are also shown in figures $6 c$ and $6 d$. In figure $6 c$, the end view of the vortex lines at $x^{+} \approx-380$ displays the inverted vortical structure; the end view of the vortex lines at $x^{+} \approx-190$ displays the regular horseshoe vortical structure in figure $6 d$. Placing the detection point farther away $\left(y^{+}=300\right.$, VISA-3) does not yield any stronger structures, as can be seen in figure 7. It is interesting, however, to note that figure 7 displays horseshoe structures whose legs originate at about $y^{+} \approx 170$ (figure $7 a$ ) and $y^{+} \approx 320$ (figure 76 ). The existence of the horseshoe vortices whose legs are located far away from the wall region suggests that the presence of a wall does not appear to be necessary for the formation of the horseshoe vortices. Figure 8 shows a side view of the same vortex lines seen in figures $5 b, 6 a$ and $7 a$. It can be seen that the horseshoe vortices are inclined at about $45^{\circ}$ to the wall. Their upper portions generally are inclined at larger angles, which is consistent with the analysis of Hama (1962) who found that the self-induced velocity of a curved vortex is maximum in the region where the curvature of the vortex line is maximum.

The second quadrant of the $\left(u^{\prime}, v^{\prime}\right)$-space, $u^{\prime}<0$ and $v^{\prime}>0$, corresponds to the ejection of low-speed fluid from the wall region. One would anticipate that motion of this kind would be induced by the horseshoe vortices. QD-2 is designed to detect such events. The vortex lines obtained from this conditional sampling technique are shown in figure 9. In the neighborhood of the detection point $(x=0, z=0)$, pronounced horseshoe-shape vortical structures are clearly discernible. The side view of the vortex lines illustrates that these vortices are again inclined at about $45^{\circ}$ to the wall. In comparison with the previous figures obtained from the variants of 
VITA (figures 3 through 8), QD-2 detects stronger horseshoe vortices. Examination of the vortex lines at all $y$-locations indicates that QD-2 does not yield the inverted horseshoe vortices observed upstream and above the regular horseshoe vortex in the VISA series.

In figure 10, a vortex filament corresponding to QD-2 is shown in the vicinity of the detection point. This vortex filament was constructed by the vortex lines which pass through a small circular region in the $(x, y)$-plane at $y^{+} \approx 55$ and $x^{+} \approx 0$. The side view of this filament again shows that the vortex is inclined at about $45^{\circ}$ to the wall. The two legs are extended toward the spanwise direction rather than pointing to the upstream direction as suggested by some models of wall-layer turbulence (Blackwelder 1978; Blackwelder \& Eckelmann 1979; Smith 1984). The top view of the filament appears to suggest that the cross section of the head region is slightly bigger than the legs, indicating that the vorticity in the head is slightly weaker than that of the legs. However, the head of this filament is located far away from the wall at approximately $y^{+}=200$, and it provides a significant vorticity perturbation in that region.

In contrast to the "hole" technique (Lu \& Willmarth 1973) used in QD-2, which detects events associated with Reynolds shear stress larger than a threshold value, QD-A and QD-B select events associated with the Reynolds shear stress in a specified range ("window"). Figure 11 shows the results obtained from using two different window sizes. By comparing figures 9 and 11, it is clear that the stronger horseshoe vortices are associated with the higher Reynolds shear stress - not a surprising result considering their induced motions.

As mentioned earlier in this section, the ensemble-averaged vorticity fields obtained from variants of the VITA technique (VISA, VISA-1,2,3) suggest that there are two distinct vortical structures associated with the sweep and ejection events. The horseshoe vortices obtained by QD-2 correspond to the latter. To investigate the vortical structures directly associated with the sweep event, QD-4 is applied to 
the data base. The vortex lines of the ensemble-averaged vorticity field obtained using QD-4 are shown in figure 12. A vortex filament near the detection point, containing the vortex lines passing through a small circular region in the $(x, y)$-plane at $y^{+} \approx 170$ and $x^{+} \approx 0$, is shown in figure 13. As anticipated from the previous results (figures 5 through 7), it displays more pronounced "inverted" horseshoe vortices associated with the sweep event. The side view of this structure (figure $12 b$ ) shows that these vortices are also inclined at about $45^{\circ}$ to the wall. Although the head of this inverted horseshoe is now located upstream of its legs and closer to the wall, the qualitative shape of the structure is strikingly similar to the "regular" horseshoe. The end and top views (figures $13 c, d$ ) suggest that the cross section of the filament in the head region is not circular but elliptical owing to the vortex stretching mechanism (see Part 1).

\section{Discussion}

The ensemble-averaged vorticity fields provide strong evidence for the connection of the bursting process with horseshoe vortices. Two different vortical structures are identified which are associated with the sweep and ejection events, respectively. Since these structures are identified by vortex lines in three- dimensional space, it is clear that the educed structure is a vortical one. Moreover, these vortical structures are important to the dynamics of the flow since they are associated with high Reynolds shear stress; figure 9 and 11 suggest that the stronger horseshoe vortices are associated with the higher Reynolds shear stress. The measurements of Willmarth \& Lu (1972) show that about $30 \%$ of the total Reynolds shear stress is due to motions in the second quadrant with local Reynolds shear stress in excess of 10 times the mean value. Recent experimental measurements by Alfredsson \& Johansson (1984) indicate that these motions contribute $20 \%$ of total Reynolds shear stress. However, this is the contribution only from the strong horseshoe 
vortices, and the total contribution from all the horseshoe vortices is expected to be much larger.

The cause of the formation of these vortical structures is not as clear as is the evidence for their existence. This is mainly because we only have static pictures. One has to follow the development of such structures dynamically to investigate the creation process. However, because the legs of the horseshoe vortices point to the spanwise direction without much elongation in the streamwise direction (pointing upstream) and because some of them are originated away from the wall (results from VISA-3 suggests as far away as $y^{+} \approx 300$ ), Taylor-Gortler-type instabilities owing to streamline curvature in the wall region (Brown \& Thomas 1977; Coles 1978) appear to be precluded as the sole source of the horseshoe vortices. It is feasible that the curvature effects amplify the streamwise vorticity wherever the correct streamwise curvature exists. Most likely, however, as pointed out in Part 1 , these vortex lines are deformed from the primary vortex lines originally parallel to the spanwise direction by random fluctuations of normal velocity component. Whenever vortex lines are deformed by a fluid motion either toward the wall or away from the wall, the presence of shear will produce both the regular and the inverted horseshoe vortices by the vortex-stretching mechanism. This can be seen from the inviscid vorticity equations,

$$
\begin{aligned}
& \frac{D \omega_{x}}{D t}=\omega_{x} \frac{\partial u}{\partial x}+\omega_{y} \frac{\partial u}{\partial y}+\omega_{z} \frac{\partial u}{\partial z} \\
& \frac{D \omega_{y}}{D t}=\omega_{x} \frac{\partial v}{\partial x}+\omega_{y} \frac{\partial v}{\partial y}+\omega_{z} \frac{\partial v}{\partial z} .
\end{aligned}
$$

Consider a perturbation associated with a localized fluid motion moving away from the wall; the only nonzero terms in the mean flow are $\omega_{z}$ and $\partial u / \partial y$. Because of the imposed perturbation, the third term in the right-hand side of (3) will create positive and negative components of the normal vorticity in the region where $\partial v / \partial z$ exists. This in turn, through the second term in (2), will create streamwise components 
of both signs. The resulting vorticity field will be that of a horseshoe vortex. The formation of an inverted horseshoe vortex can be explained similarly by considering a localized fluid motion moving toward the wall. Thus, the vortex stretching terms provide the proper mechanism for the formation of the horseshoe vortices (the regular as well as the inverted one). The final angle formed by these stretched vortices is determined by a balance between the shearing and stretching action, which is described in Part 1 (Moin \& Kim 1984). It is also interesting to note that from the third term in (2), the wall-layer streaks in the wall region (the streaky structures create locally a high $\partial u / \partial z$ ) would produce vorticity of opposite sign to that of the vorticity of the streamwise vortices that might have produced the streaks.

The stretching process just described plus the fact that the formation of some of the horseshoe vortices is begun far away from the wall region leads us to the conjecture that these vortices (the regular as well as the inverted one) exist in all shear flows irrespective of the presence of a wall. Whether these vortical structures play a major role in the dynamics of the flow may be flow-dependent. For the wallbounded shear flow considered here, these are the important structures since they have a significant contribution to Reynolds shear stress, and hence to turbulent energy production.

The ensemble-averaged vortical patterns are inclined at about $45^{\circ}$ to the wall - the same angle formed by most of the instantaneous vortex lines, as observed in Part 1. In figure $9 b$, it can be seen that the heads of the vortex lines form an envelope with an angle that is smaller than $45^{\circ}$ at the upstream side (about $20^{\circ}$ ). Head \& Bandyopadhyay (1981) observed that hairpins were occasionally produced in a regular sequence such that the envelope of their tips formed a straight line inclined at about $20^{\circ}$ to the wall (figures 13 and 14 of Head \& Bandyopadhyay). This may also be related to the large-scale structure reported by Brown \& Thomas 
(1977), as pointed out by Head \& Bandyopadhyay, although the present work deals with a turbulent channel flow instead of the boundary layer of Brown \& Thomas.

The ensemble-averaged streamwise velocity profiles associated with the vortical structures are shown in figure 14. The dashed lines represents the time-averaged mean velocity, and the solid circles denote the ensemble-averaged profile from QD4 (associated with the inverted horseshoe vortex and the sweep event); the open circles denote the profiles obtained by using QD-2 (associated with the regular horseshoe vortex and the ejection event). Both profiles are obtained at $x^{+} \approx 120$ downstream of the detection point, and they show pronounced excess and defect in the streamwise velocity associated with the sweep and ejection events, respectively. A three-dimensional display of these vortices with the corresponding ensembleaveraged streamwise velocity profiles is shown in figure 15 .

The educed structures presented in this paper are obtained from the ensembleaverage fields. These results, therefore, represent an average shape of the unsteady vortical structures in turbulent channel flow. As shown in Part 1, an instantaneous structure is not as symmetric and smooth as the ensemble-averaged one. In fact it can be argued that the educed structure is totally an artifice of an ensembleaveraging process. For example, ensemble-averaging can yield a pair of counterrotating vortices as the dominant structures associated with a given event, if the conditional sampling technique detects with equal probability a single isolated vortex of one sign to the right of the detection point and another of opposite sign to the left. To investigate whether the instantaneous detected structure has a horseshoe shape, the vortex lines corresponding to a single realization are examined. An example of instantaneous vortex lines from a single realization drawn in the area near a QD-2 detection point, is shown in figure 16. The vortex lines in figure $16 a$ are drawn the same way as the ones in figure $9 a$; the only difference is that the lines in figure $9 a$ are ensemble-averaged, whereas the lines in figure $16 a$ are the instantaneous lines. Figure $16 a$ (and many other instantaneous pictures) clearly 
shows several horseshoe-shaped vortex lines in the vicinity of the detection point and demonstrates that the conditional sampling technique detects horseshoe vortices and that the averaged pattern is indeed representative of the instantaneous structures and not the result of a spurious result of the ensemble-averaging process. A vortex filament in the vicinity of the detection point is shown in figure $16 b$.

\section{Summary and conclusions}

In order to investigate the organized vortical structures associated with the bursting process, several conditional sampling techniques are applied to a data base generated by large-eddy simulation of turbulent channel flow. The vortical structures are identified by examining vortex lines of the three-dimensional ensemble-averaged vorticity field. These ensemble-averaged vortical structures display the well-organized horseshoe vortices. VISA, which is adapted from the original VITA (Blackwelder \& Kaplan 1976) to obtain a spatial rather than temporal structure, does not yield well-defined horseshoe vortices. Several variants of the VITA techniques are used in the present work. It is found that there exist two distinct horseshoe-shaped vortices associated with the bursting event. The regular horseshoe vortex is associated with the ejection (of low-speed fluid in the wall-region) event and the "inverted" horseshoe vortex with the sweep event (high-speed fluid moving toward the wall region).

The QD-2 and QD-4 averaging techniques, which directly sample the ejection [the second quadrant in the $\left(u^{\prime}, v^{\prime}\right)$-space] and sweep events (the fourth quadrant), respectively, provides stronger indication of horseshoe vortices. The ensembleaveraged vortical structure has generally the same characteristics as the instantaneous structures (Moin \& Kim 1984): they are inclined at about $45^{\circ}$ and their legs are extended in the spanwise directions without much elongation in the streamwise direction. The conditional sampling criteria are triggered by high Reynolds shear 
stress indicating that these structures make a significant contribution to turbulent energy production.

It was shown that the horseshoe vortices are also formed in the regions away from the wall from deformation of the primary vortex lines. This leads us to conjecture that horseshoe (or hairpin) vortices are not peculiar to wall-bounded flows but are characteristic of all shear flows. For the wall-bounded shear flows considered here, these are the dominant structures in the sense that they survive the ensembleaveraging process (repeatable) and are associated with high Reynolds shear stress (energetic).

The authors are grateful to Drs. A. Leonard and R. S. Rogallo for helpful comments on a draft of this manuscript. 
Table 1

Conditionl sampling techniques

\begin{tabular}{|c|c|}
\hline VISA & $\begin{array}{l}\widehat{u}\left(x, y, z, t_{0}, L\right)=\frac{1}{L} \int_{x-\frac{L}{2}}^{x+\frac{L}{2}} u\left(\xi, y, z, t_{0}\right) d \xi \\
\widehat{v a r}=\widehat{u^{2}}-\widehat{u}^{2} \\
\begin{aligned} D(\xi)=1, \text { if } \widehat{v a r}>k u_{r m s}^{2} \text { and } \frac{\partial u}{\partial x}<0 \\
\quad=0, \text { otherwise }\end{aligned} \\
<u\left(x, y, z, t_{0}\right)>=\frac{1}{N} \sum_{j=1}^{N} u\left(x+\xi_{j}, y, z, t_{0}\right) \\
\text { where } k=1.2, L^{+}=500 \text { and } \\
\text { the detection point at } y^{+}=21\end{array}$ \\
\hline VISA-1 & $\begin{array}{l}\text { Same as VISA except that: } \\
\begin{aligned} D(\xi)=1, \text { if } \widehat{v a r}>k u_{r m s}^{2} \text { and } \frac{\partial u}{\partial x}>0 \\
\quad=0, \text { otherwise }\end{aligned} \\
\text { Detection point at } y^{+}=100\end{array}$ \\
\hline VISA-2 & Same as VISA-1 except $y^{+}=200$ \\
\hline VISA-3 & Same as VISA-1 except $y^{+}=300$ \\
\hline QD-2 & 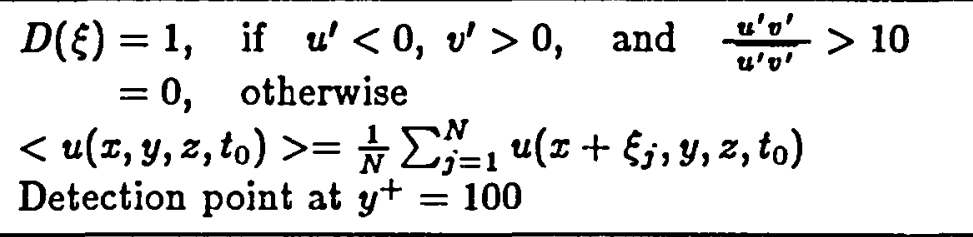 \\
\hline QD-4 & $\begin{array}{l}D(\xi)=1, \text { if } u^{\prime}>0, v^{\prime}<0, \text { and } \frac{u^{\prime} v^{\prime}}{u^{\prime} v^{\prime}}>10 \\
\quad=0, \text { otherwise }\end{array}$ \\
\hline QD-A & $\begin{array}{l}\text { Same as QD-2 except that: } \\
\begin{aligned} D(\xi) & =1, \text { if } u^{\prime}<0, v^{\prime}>0, \text { and } 0<\frac{u^{\prime} v^{\prime}}{u^{\prime} v^{\prime}}<1 \\
& =0, \text { otherwise }\end{aligned}\end{array}$ \\
\hline QD-B & $\begin{array}{l}\text { Same as QD-2 except that: } \\
\begin{aligned} D(\xi) & =1, \quad \text { if } u^{\prime}<0, v^{\prime}>0, \text { and } 1<\frac{u^{\prime} v^{\prime}}{u^{\prime} v^{\prime}}<2 \\
& =0, \text { otherwise }\end{aligned}\end{array}$ \\
\hline
\end{tabular}




\section{REFERENCES}

Alfredsson, P. H. \& Johansson, A. V. 1984 On the detection of turbulence-generating events. J. Fluid Mech. 139, 325.

BLACK WELDER, R. F. 1978 The bursting process in turbulent boundary layers. In Coherent Structure of Turbulent Boundary Layers (ed. C. R. Smith \& D. E. Abbott), p.211. AFOSR/Lehigh University Workshop, Dept. Mech. Engng. \& Mech., Bethlehem, Pennsylvania.

BLACKWELDER, R. F. \& KAPLAN, R. E. 1976 On the wall structure of the turbulent boundary layer. J. Fluid Mech. 76, 89.

BLACKWELDER, R. F. \& ECKELMANN, H. 1979 Streamwise vortices associated with the bursting phenomenon. J. Fluid Mech. 94, 577.

Brown, G. L. \& Thomas, A. S. W. 1977 Large structure in a turbulent boundary layer. Phys. Fluids 20, S243.

Chen, C. H. P. \& Blackwelder, R. F. 1978 Large scale motion in a turbulent boundary layer: a study using temperature contamination. J. Fluid Mech. 89, 1.

Coles, D. 1978 A model for flow in the viscous sublayer. In Coherent Structure of Turbulent Boundary Layers (ed. C. R. Smith \& D. E. Abbott), p.462. AFOSR/Lehigh University Workshop, Dept. Mech. Engng. \& Mech., Bethlehem, Pennsylvania.

Corino, E. R. \& BRodKey R. S. 1969 A visual investigation of the wall region in turbulent flow. J. Fluid Mech. 37, 1.

Hama, F. R. 1962 Progressive deformation of a curved vortex filament by its own induction. Phys. Fluids 5, 1156.

HEAD, M. R. \& BANDYOPADHYAY, P. 1981 New aspects of turbulent boundary layer structure. J. Fluid Mech. 107, 297.

KIM, J. 1983 On the structure of wall-bounded turbulent flows. Phys. Fluids 26, 2088.

KIM, J. 1984 Turbulence structures associated with the bursting event, Phys. Fluids, in press; see also NASA TM-85944.

KIM, H. T., KLINE, S. J., \& REYNolds, W. C. 1971 The production of turbulence near a smooth wall in a turbulent boundary layer. J. Fluid Mech. 50, 133. 
KLINE, S. J. 1978 The role of visualization in the study of the structure of the turbulent boundary layer. In Coherent Structure of Turbulent Boundary Layers (ed. C. R. Smith \& D. E. Abbott), p.1. AFOSR/Lehigh University Workshop, Dept. Mech. Engng. \& Mech., Bethlehem, Pennsylvania.

Kline, S. J., Reynolds, W. C., Schraub, F. A., \& Runstadler, P. W. 1967 The structure of turbulent boundary layers. J. Fluid Mech. 30, 741.

LEONARD, A. 1980 Vortex simulation of three-dimensional spotlike disturbances in a laminar boundary layer. Turbulent Shear Flows 2, Berlin Heidelberg: Springer-Verlag, 67.

LU, S. S. \& Willmarth, W. W. 1973 Measurements of the structure of the Reynolds stress in a turbulent boundary layer. J. Flu:d Mech. 60, 481.

MoIN, P. 1984 Probing turbulence via large eddy simulation. AIAA Paper 840174.

MoIN, P. \& KIM, J. 1982 Numerical investigation of turbulent channel flow. J. Fluid Mech. 118, 341.

MoIN, P. \& KIM, J. 1984 The structure of the vorticity field in turbulent channel flow. Part 1. Analysis of instantaneous fields and statistical correlations. Submitted to J. Fluid Mech.

SMITH, C. R. 1984 A synthesized model of the near-wall behavior in turbulent boundary layers. In Proc. of Eighth Symposium on Turbulence (ed. G. K. Patterson \& J. L. Zakin). Dept. of Chem. Engng., University of Missouri-Rolla.

Wallace, J. M., ECKelmanN, H \& Brodkey, R. S. 1972 The wall region in turbulent shear flow. J. Fluid Mech. 54, 39.

Willmarth, W. W. \& TU, B. J. 1967 Structure of turbulence in the boundary layer near the wall. Phys. Fluids 10, S134.

Willmarth, W. W. \& LU, S. S. 1972 Structure of the Reynolds stress near the wall. J. Fluid Mech. 55, 65. 

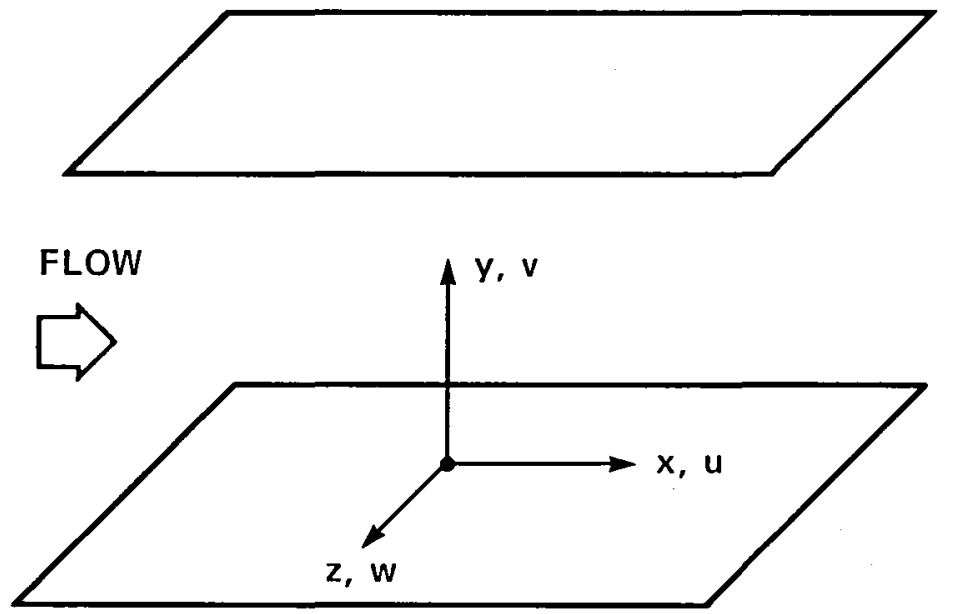

Figure 1. Coordinate system in channel. 


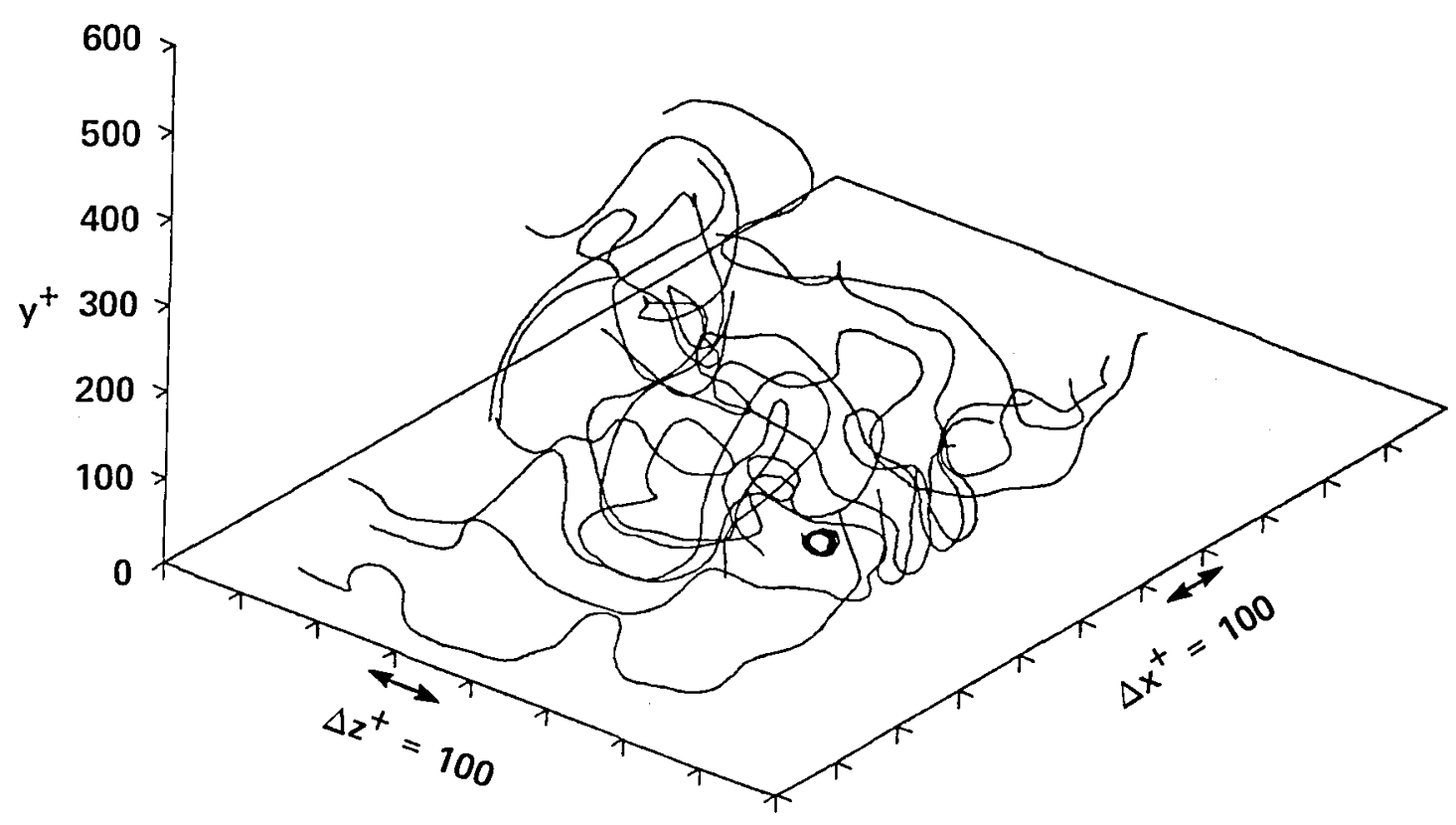

Figure 2. Randomly chosen instantaneous vortex lines in a turbulent flow. 

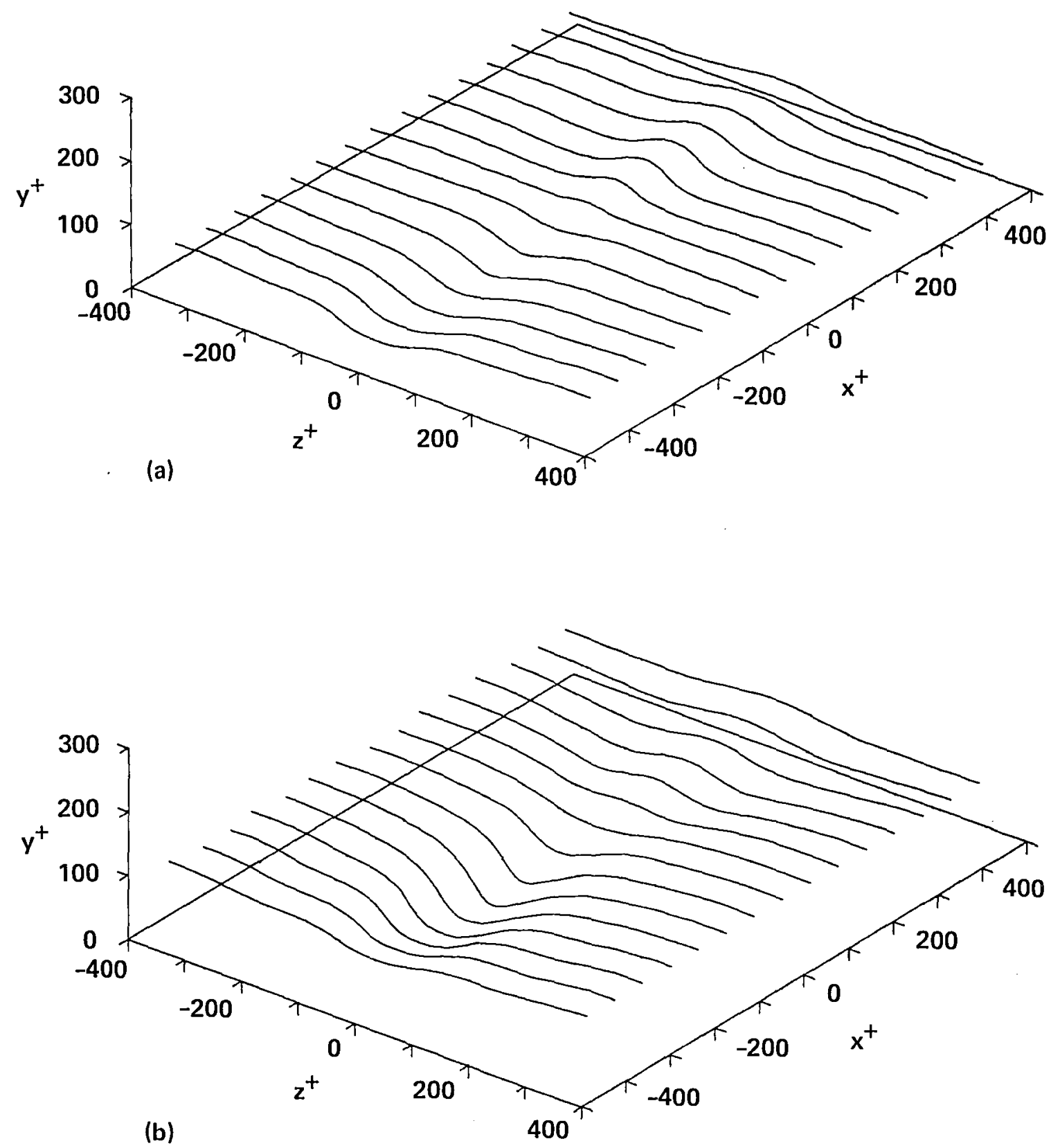

Figure 3. Vortex lines from VISA in the vicinity of the detection point: (a) lines that approach $y^{+} \approx 55$ as $z \rightarrow \infty$; (b) lines that approach $y^{+} \approx 106$ as $z \rightarrow \infty$. 


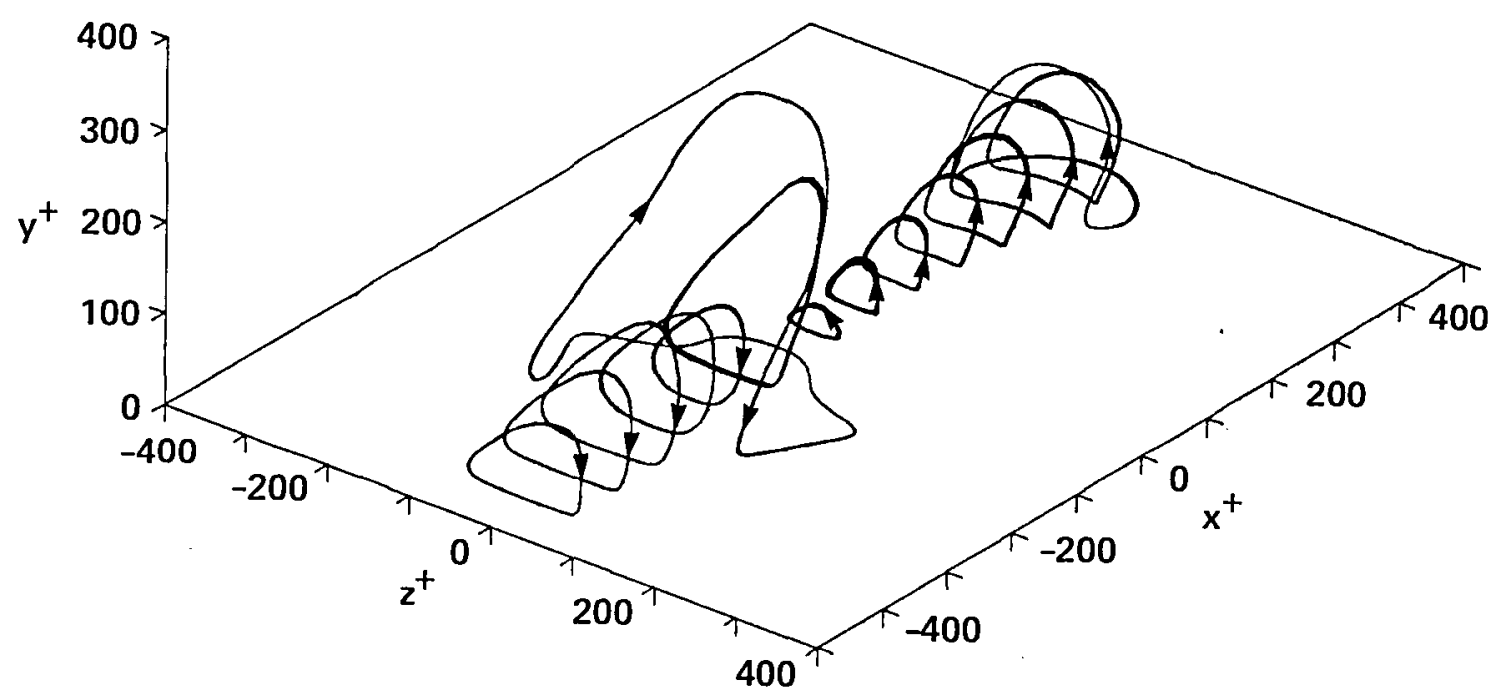

Figure 4. Vortex lines corresponding to the perturbation vorticity field. The lines shown are those that pass through the point of maximum perturbation vorticity for a given streamwise location. 

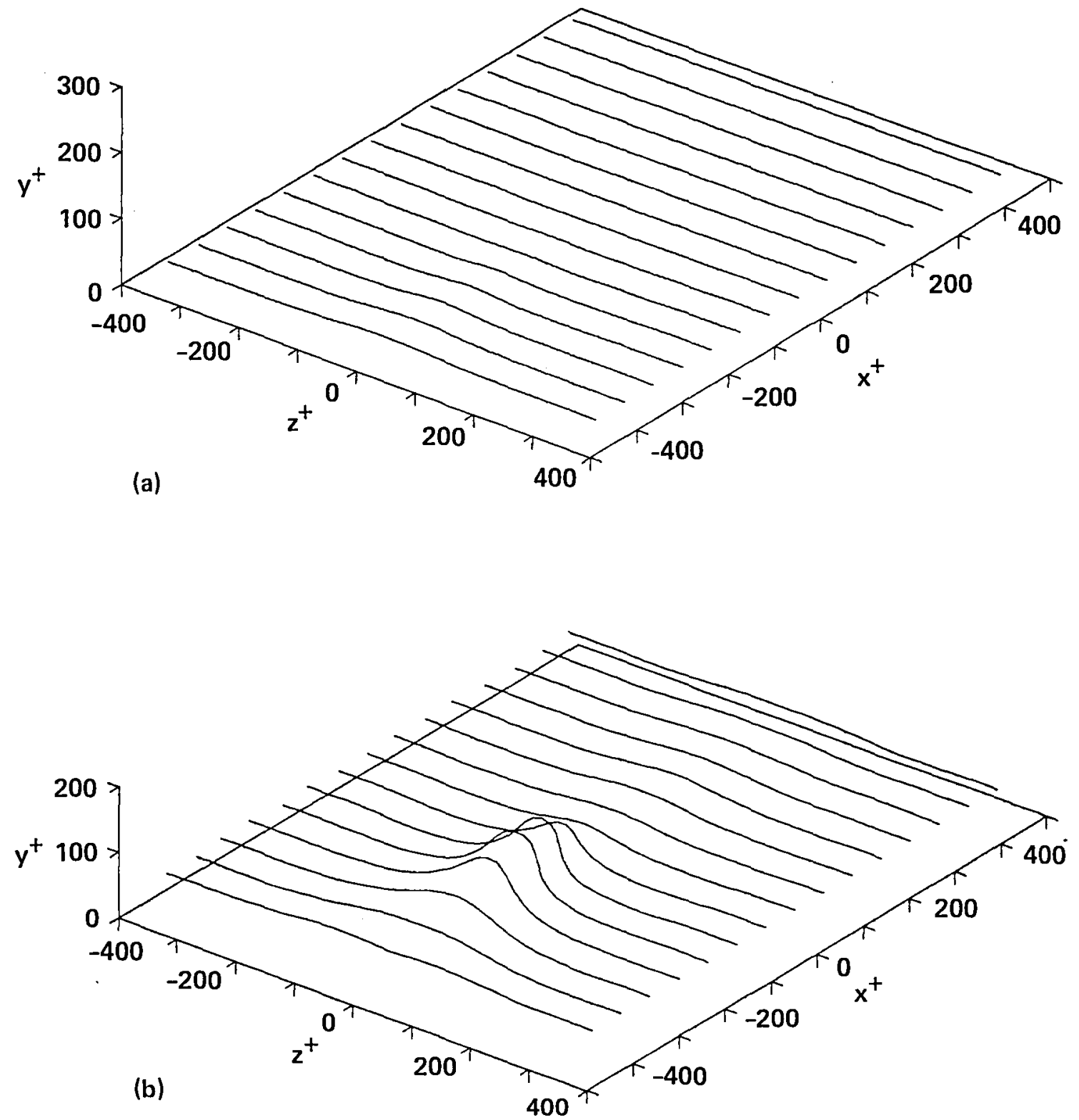

Figure 5. Vortex lines from VISA-1 in the vicinity of the detection point: (a) lines that approach $y^{+} \approx 20$ as $z \rightarrow \infty$; (b) lines that approach $y^{+} \approx 55$. 

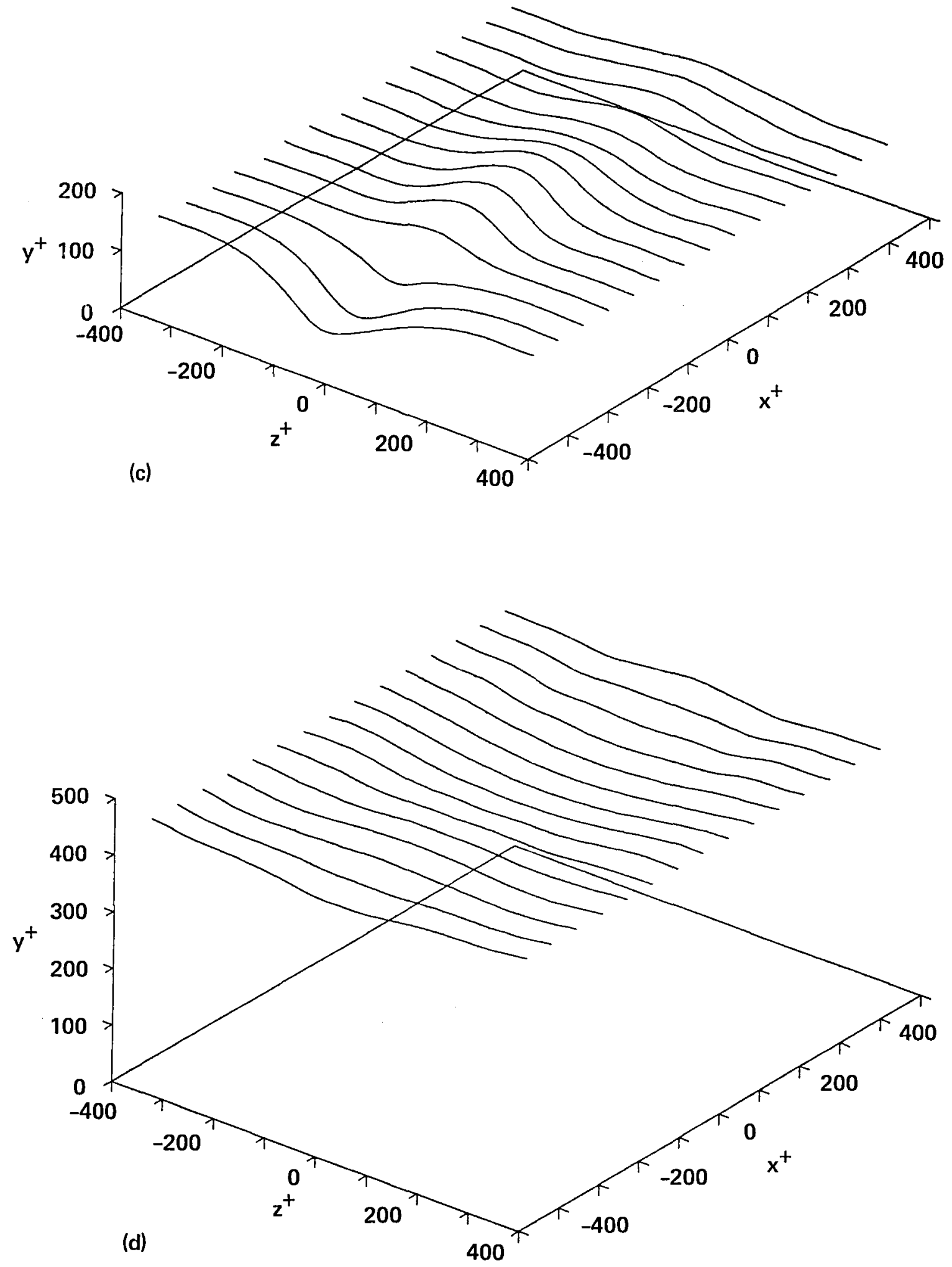

Figure 5. Concluded. (c) lines that approach $y^{+} \approx 143$; (d) lines that approach $y^{+} \approx 300$. 

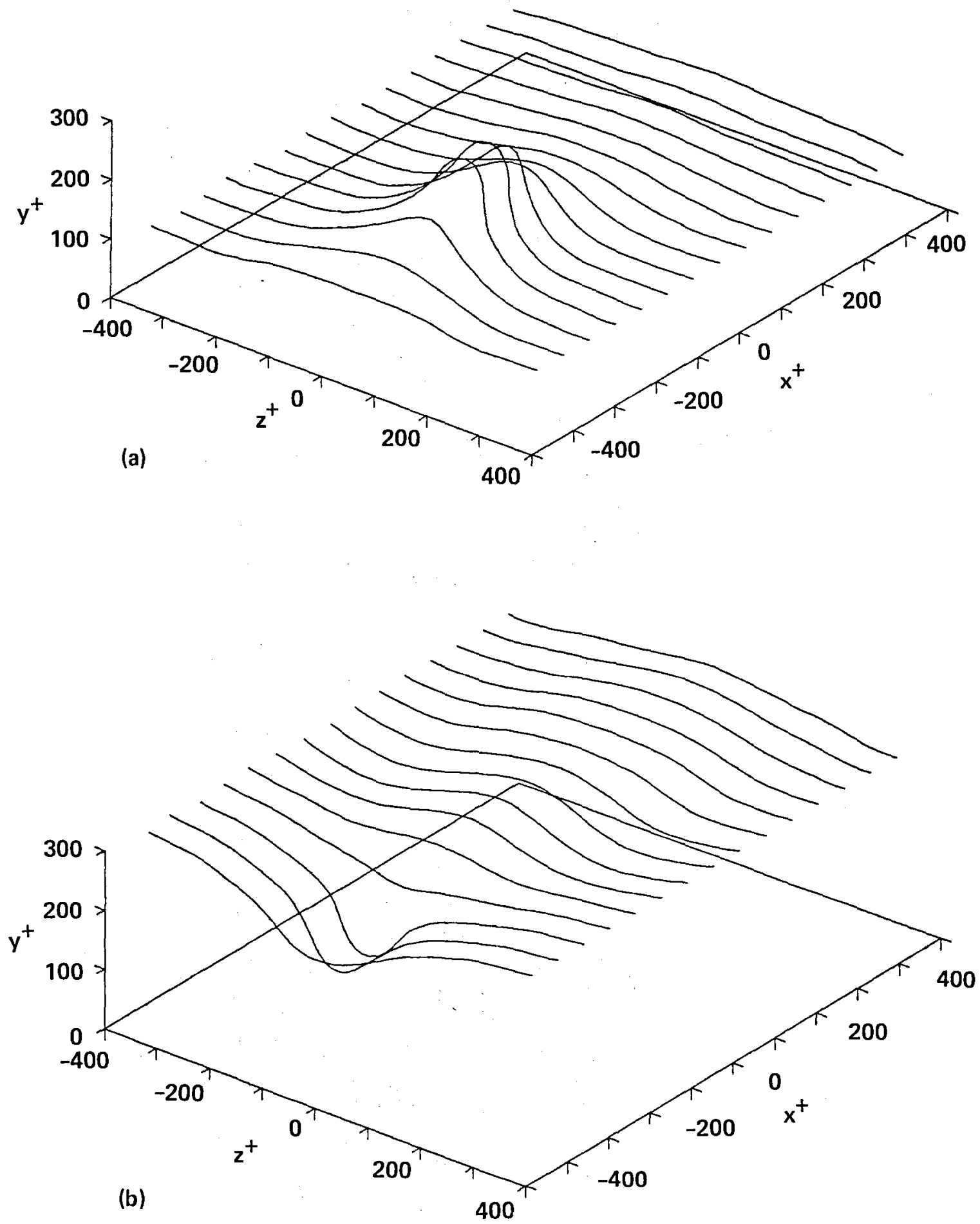

Figure 6. Vortex lines from VISA-2 in the vicinity of the detection point: (a) lines that approach $y^{+} \approx 106$ as $z \rightarrow \infty$; (b) lines that approach $y^{+} \approx 320$. 

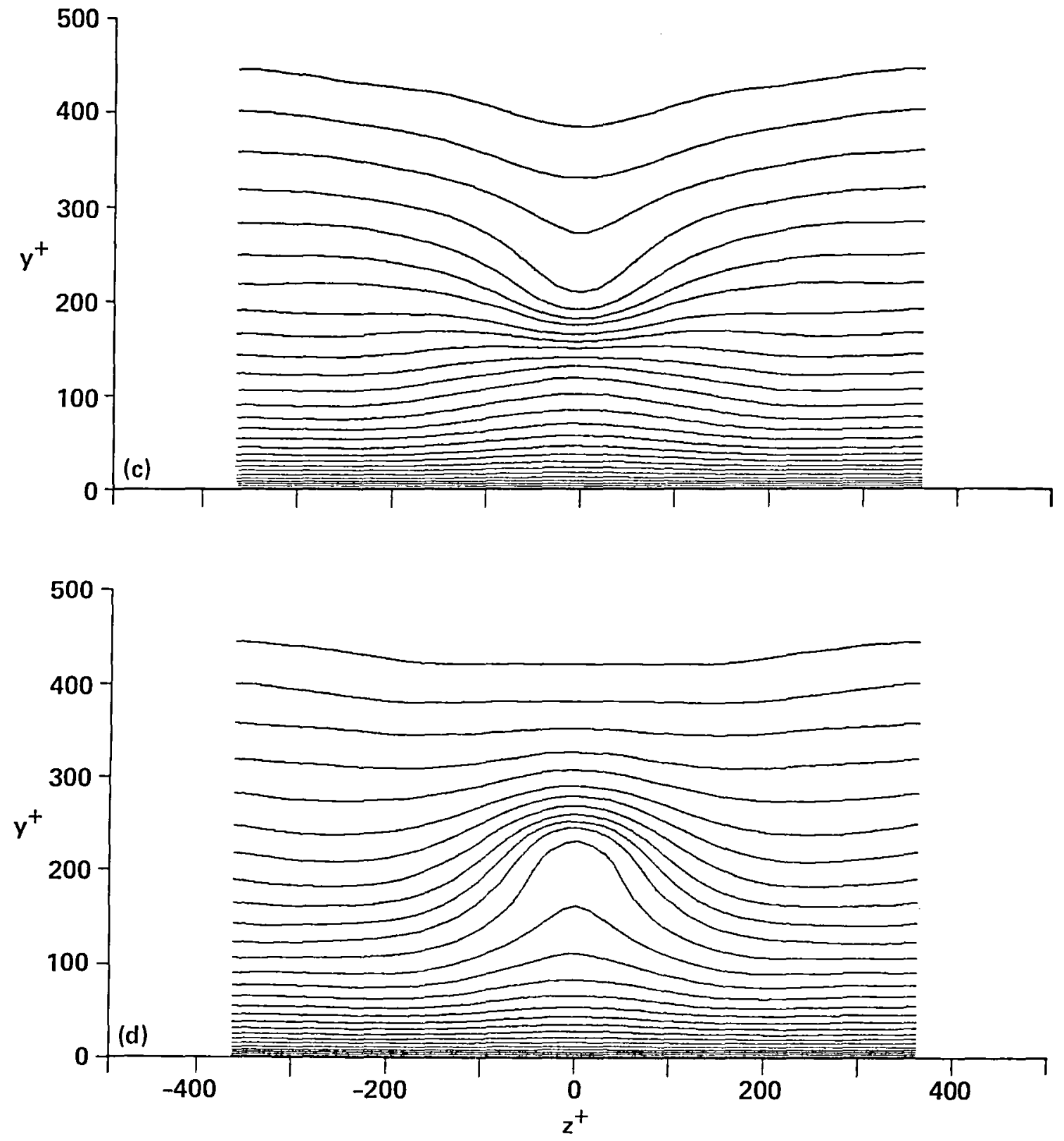

Figure 6. Concluded. (c) end view of the vortex lines at $x^{+} \approx-380$ for several $y$-locations; (d) end view of the vortex lines at $x^{+} \approx-190$. 

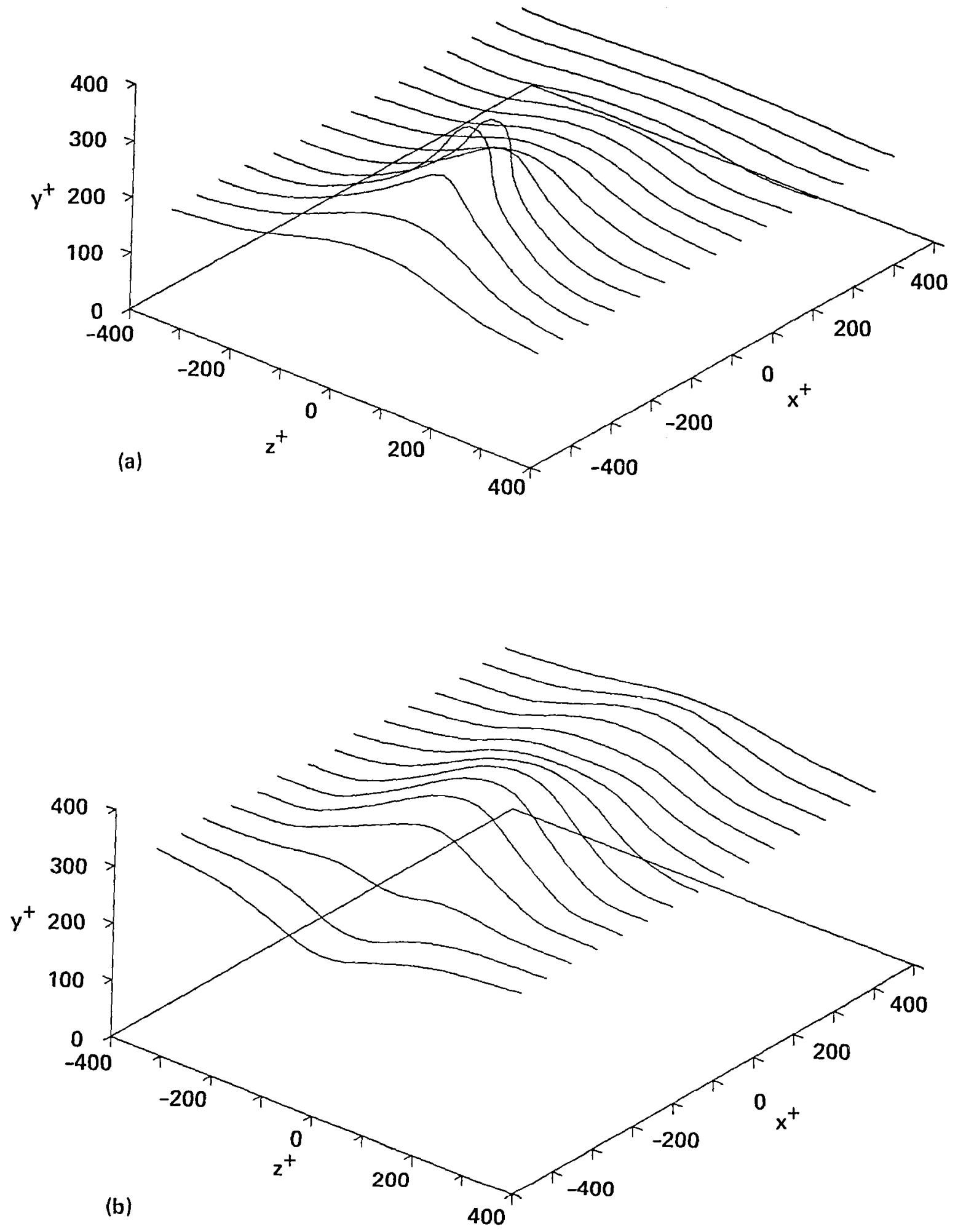

Figure 7. Vortex lines from VISA-3 in the vicinity of the detection point: (a) lines that approach $y^{+} \approx 170$ as $z \rightarrow \infty$; (b) lines that approach $y^{+} \approx 320$. 

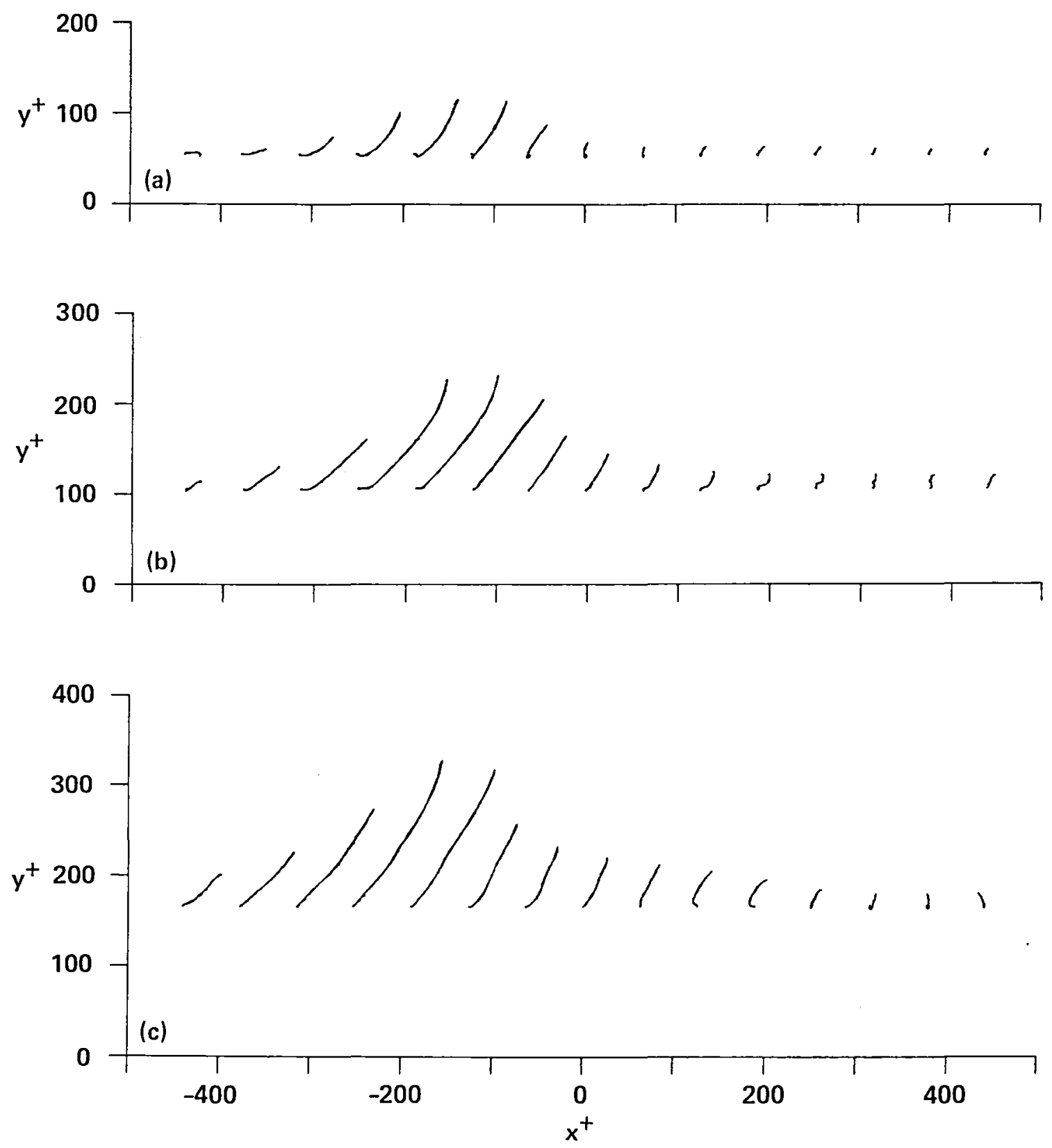

Figure 8. Side view of the vortex lines: (a) VISA-1; (b) VISA-2; (c) VISA-3. 

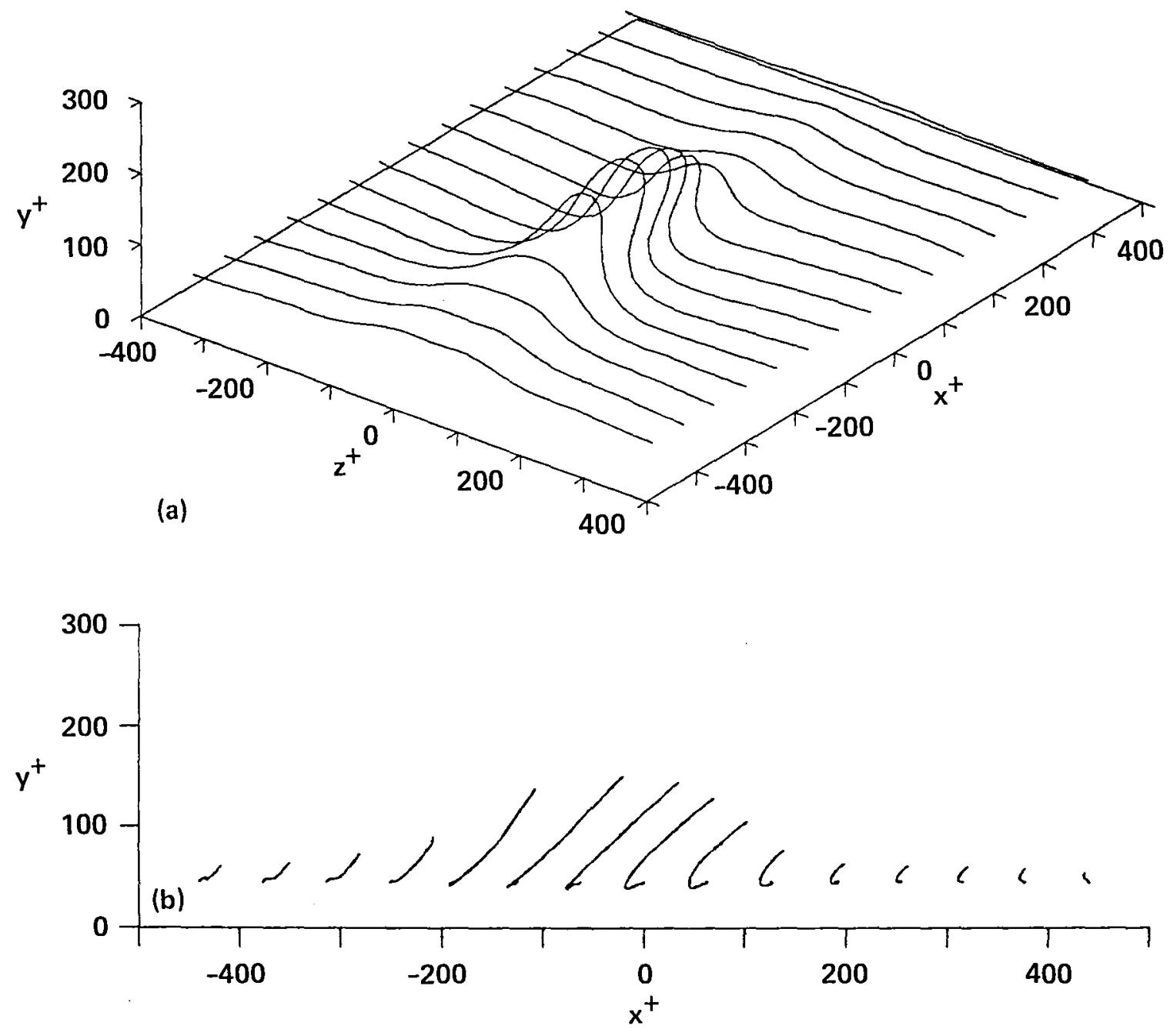

Figure 9. Vortex lines from QD-2. The lines shown are those that approach $y^{+} \approx 55$ as $z \rightarrow \infty$ : (a) oblique view; (b) side view. 

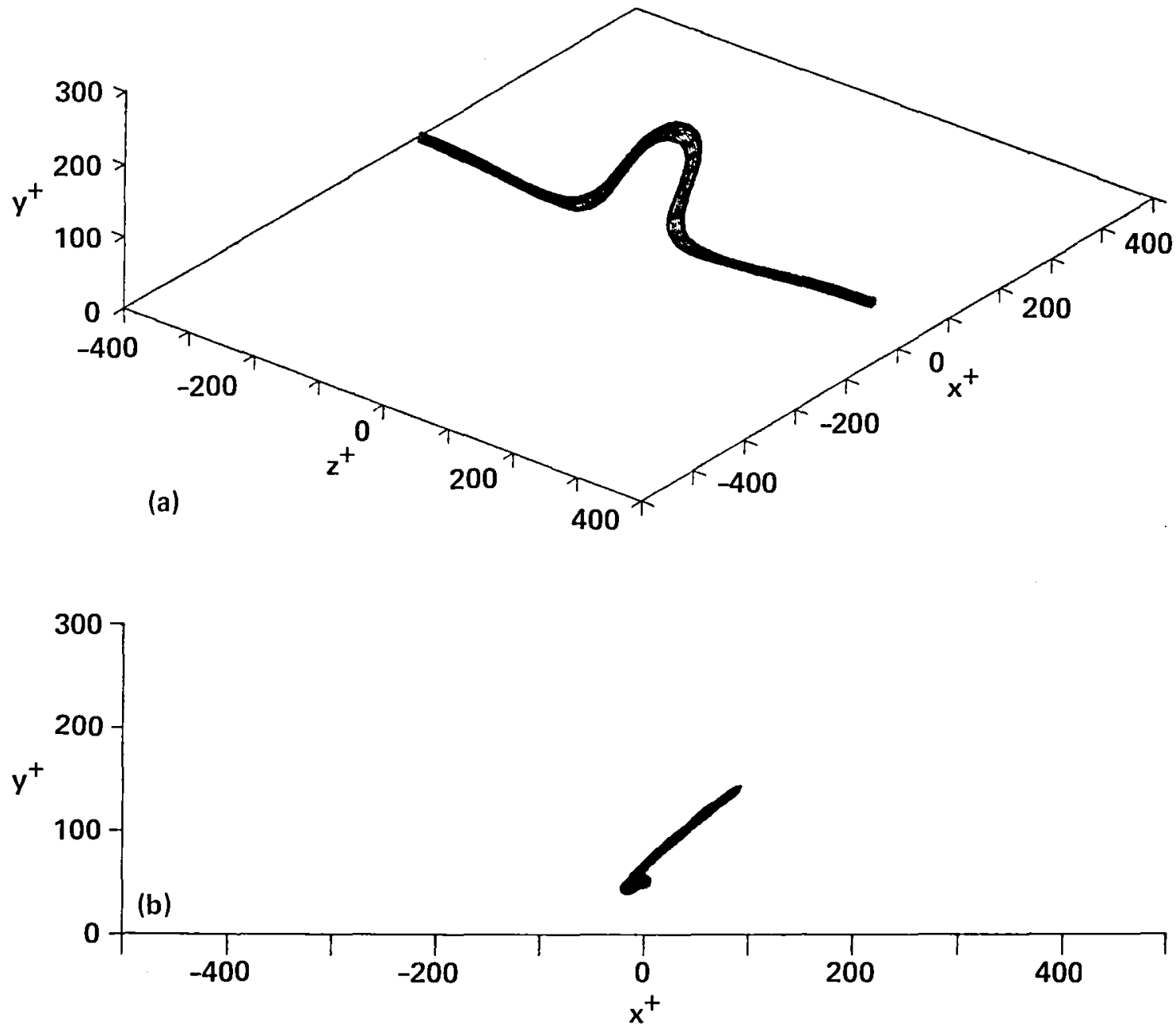

Figure 10. A vortex filament in the vicinity of the detection point from QD-2:

(a) oblique view; (b) side view. 

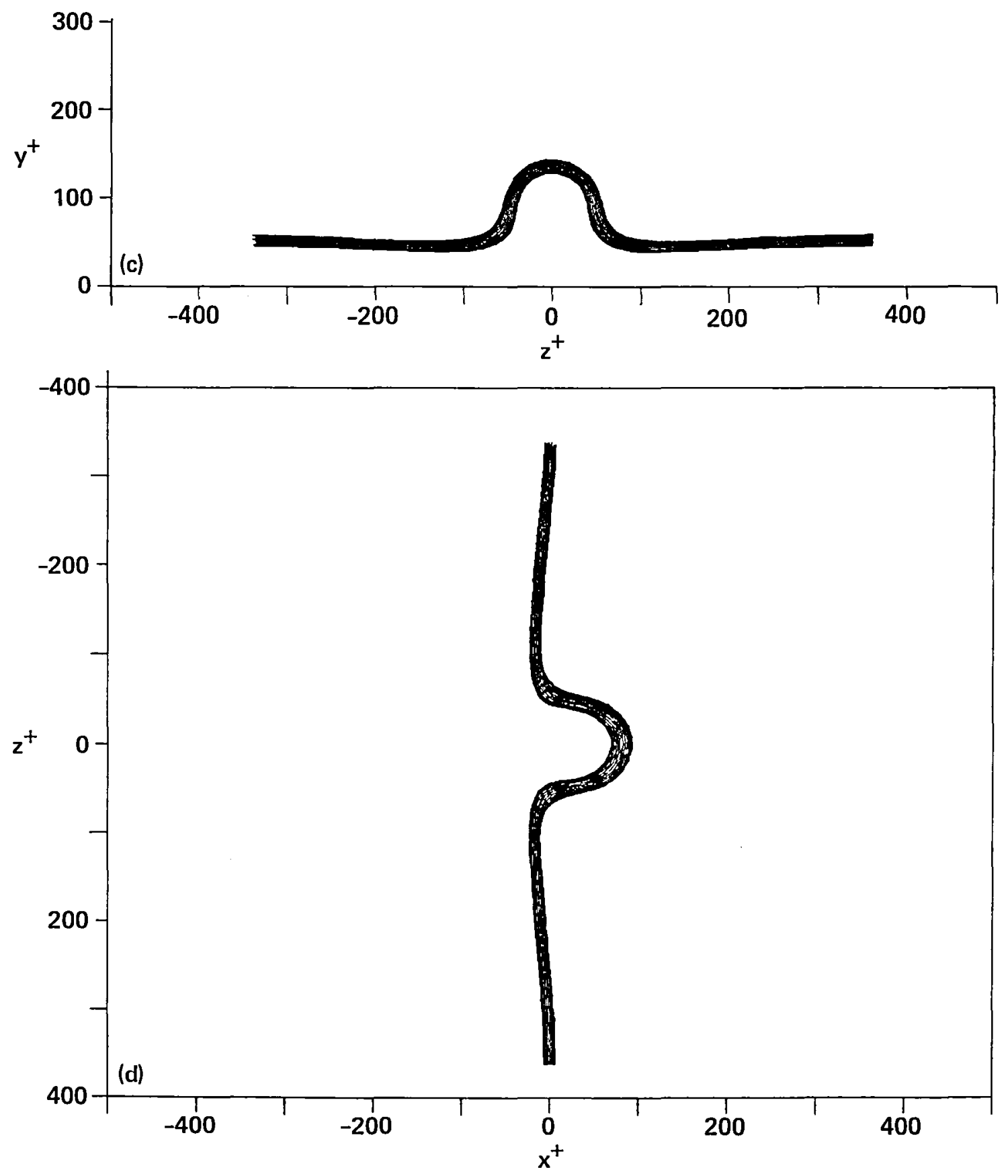

Figure 10. Concluded. (c) end view; (d) top view. 

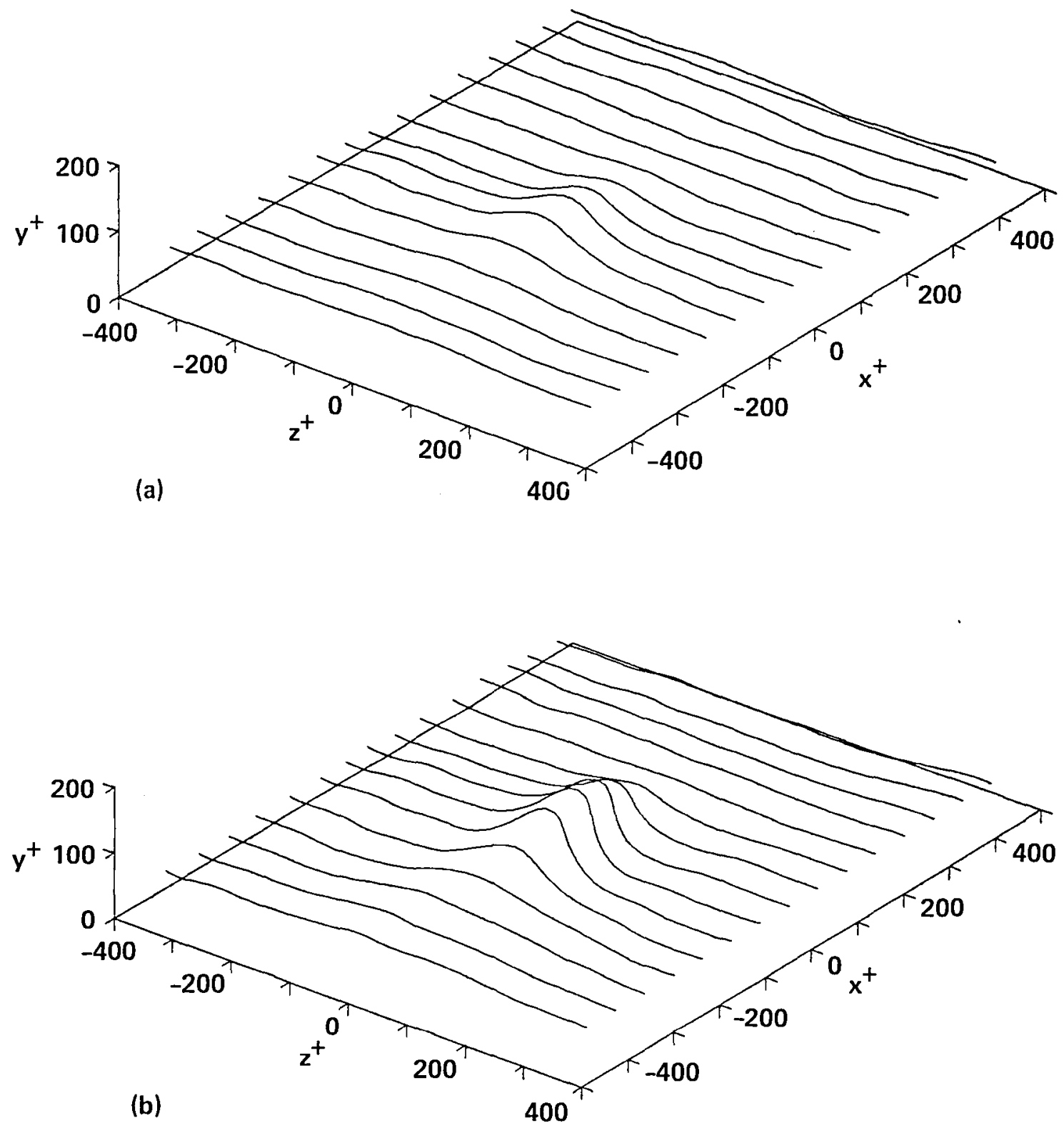

Figure 11. Vortex lines from (a) QD-A and (b) QD-B. The lines shown are those that approach $y^{+} \approx 55$ as $z \rightarrow \infty$. 

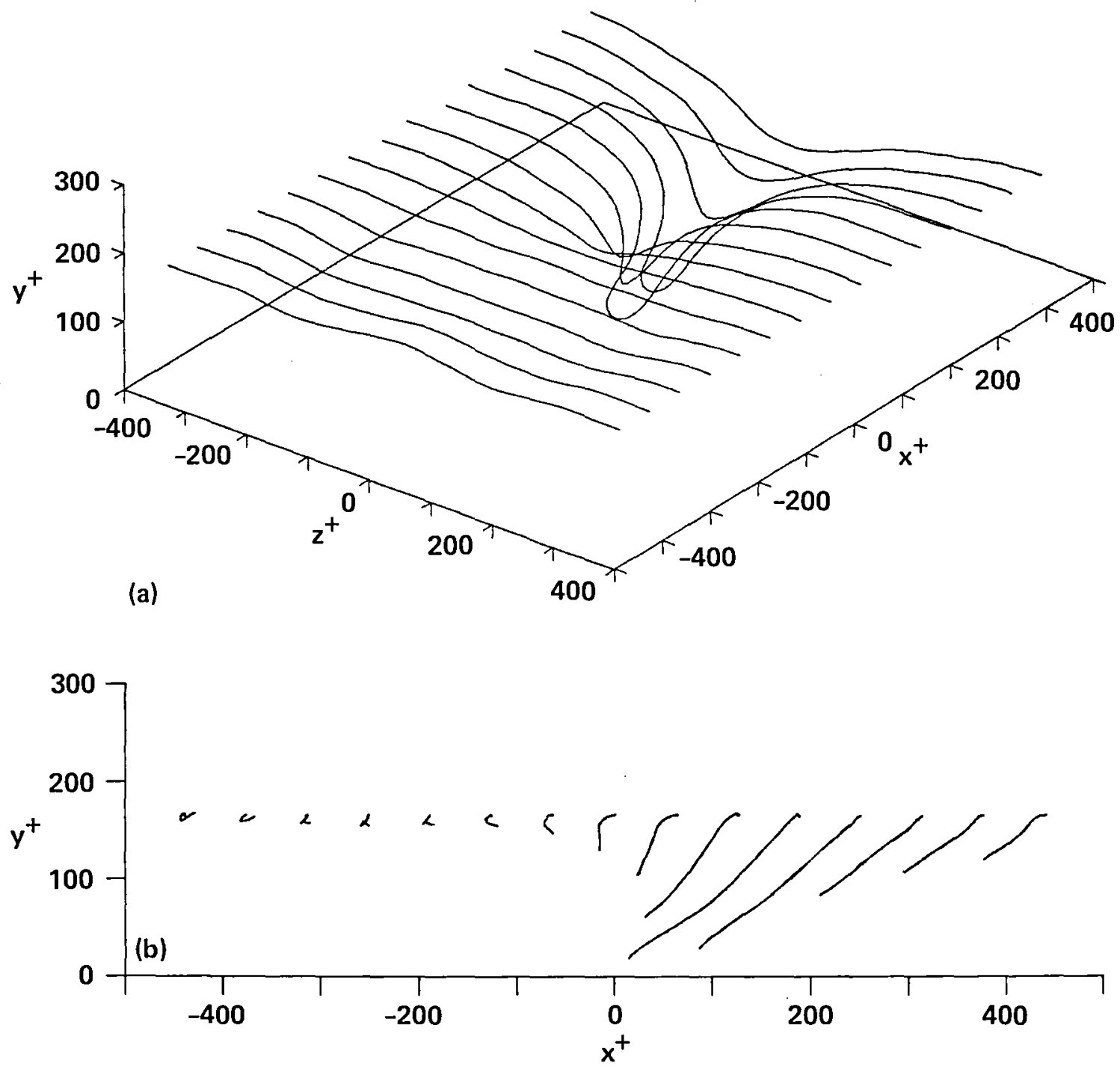

Figure 12. Vortex lines from QD-4. The lines shown are those that approach $y^{+} \approx 170$ as $z \rightarrow \infty$; (a) oblique view; (b) side view. 

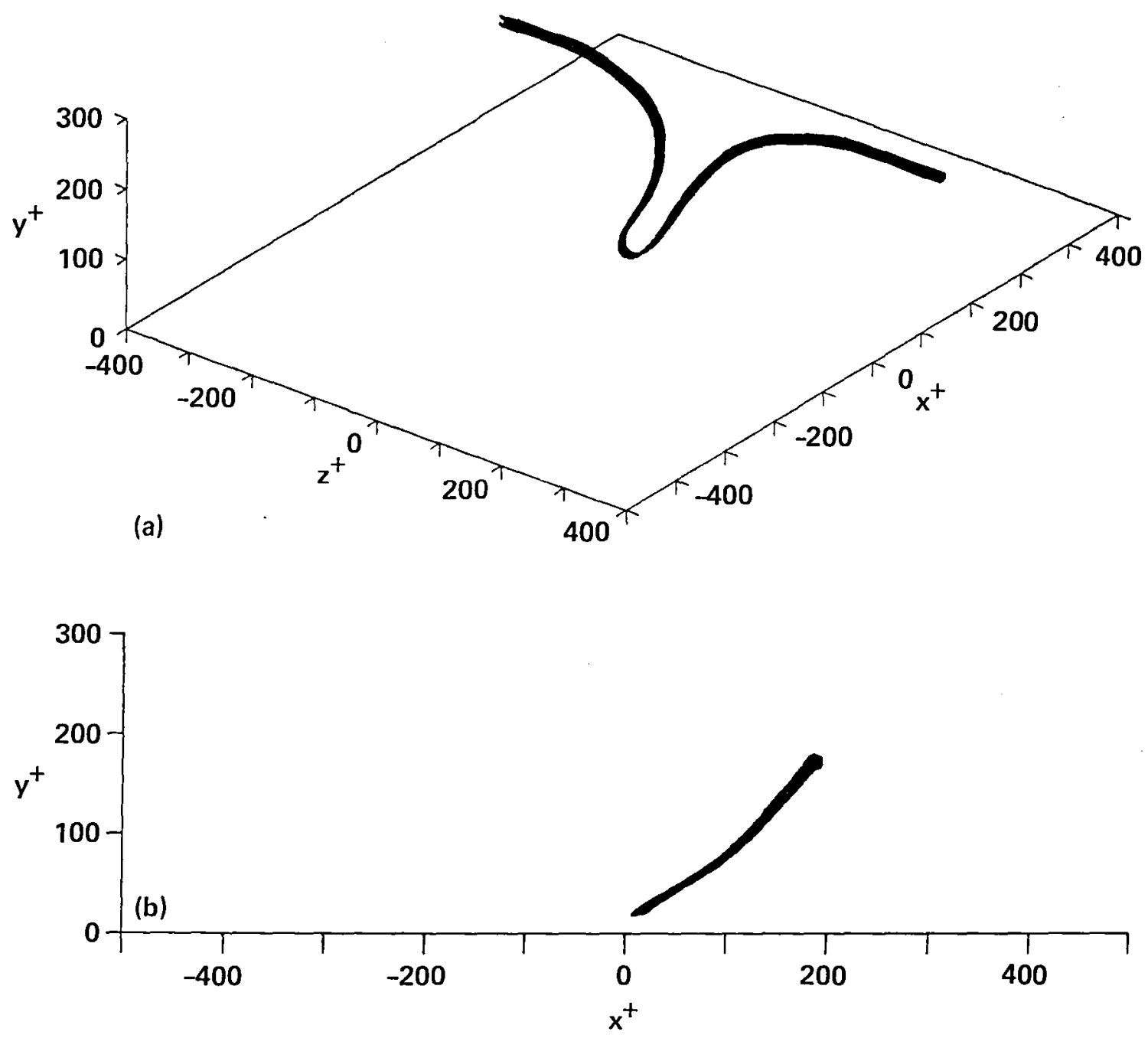

Figure 13. A vortex filament in the vicinity of the detection point from QD-4:

(a) oblique view; (b) side view. 

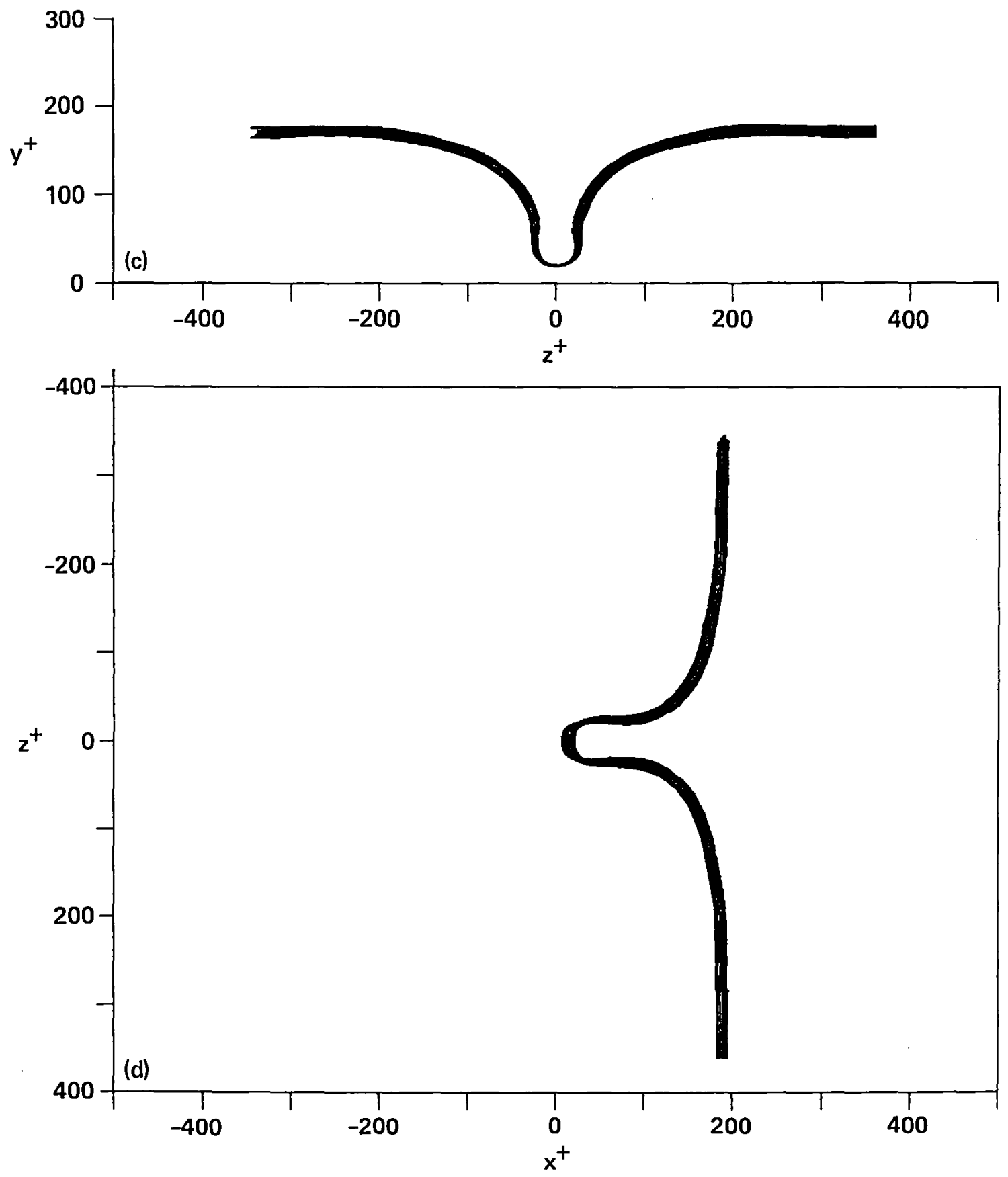

Figure 13. Concluded. (c) end view; (d) top view. 


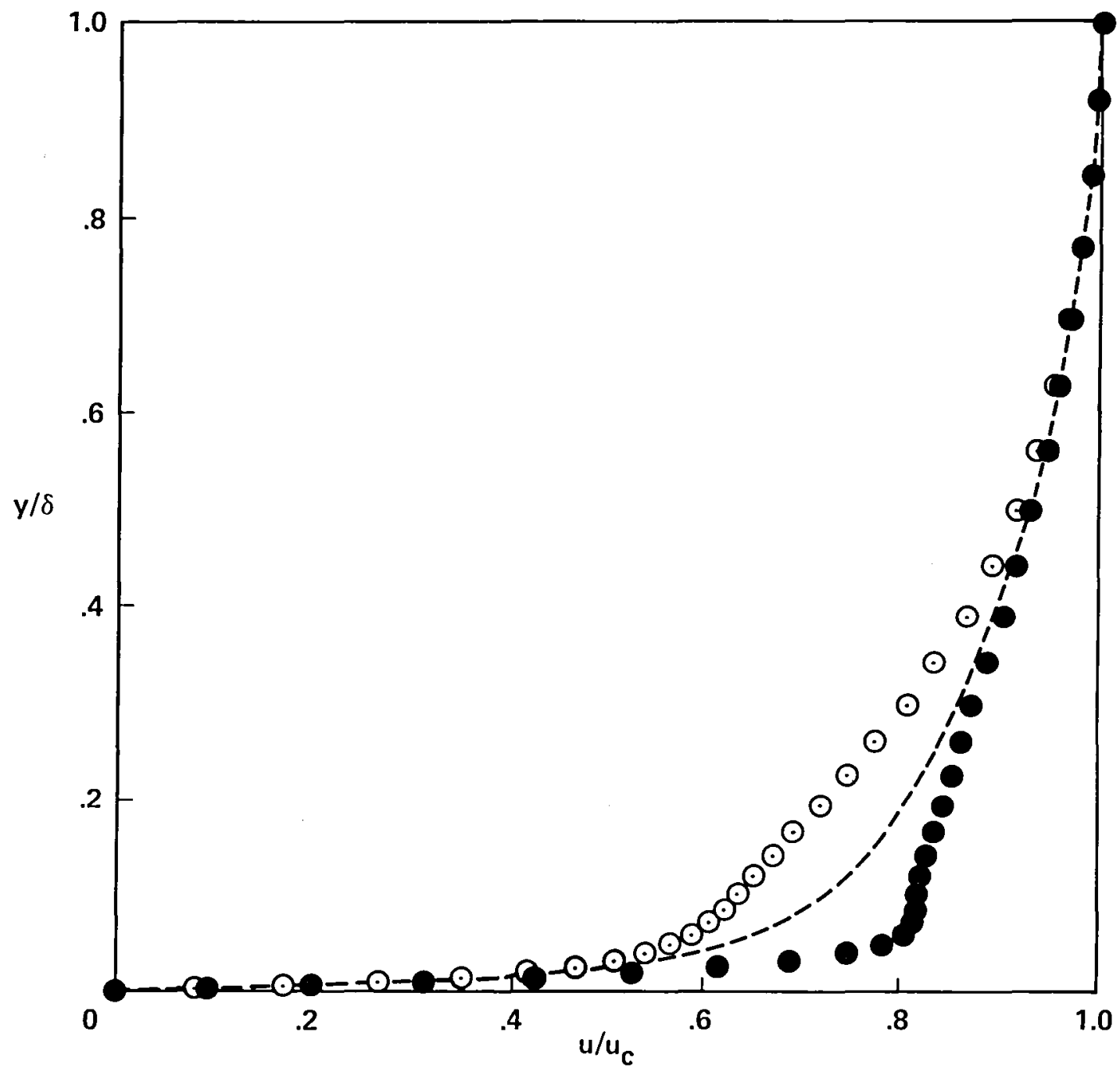

Figure 14. Mean and ensemble-averaged streamwise velocity profiles:० $\circ, \mathrm{QD}-$ $2 ; \bullet \bullet \bullet, Q D-4 ;-\cdots-$, mean. 

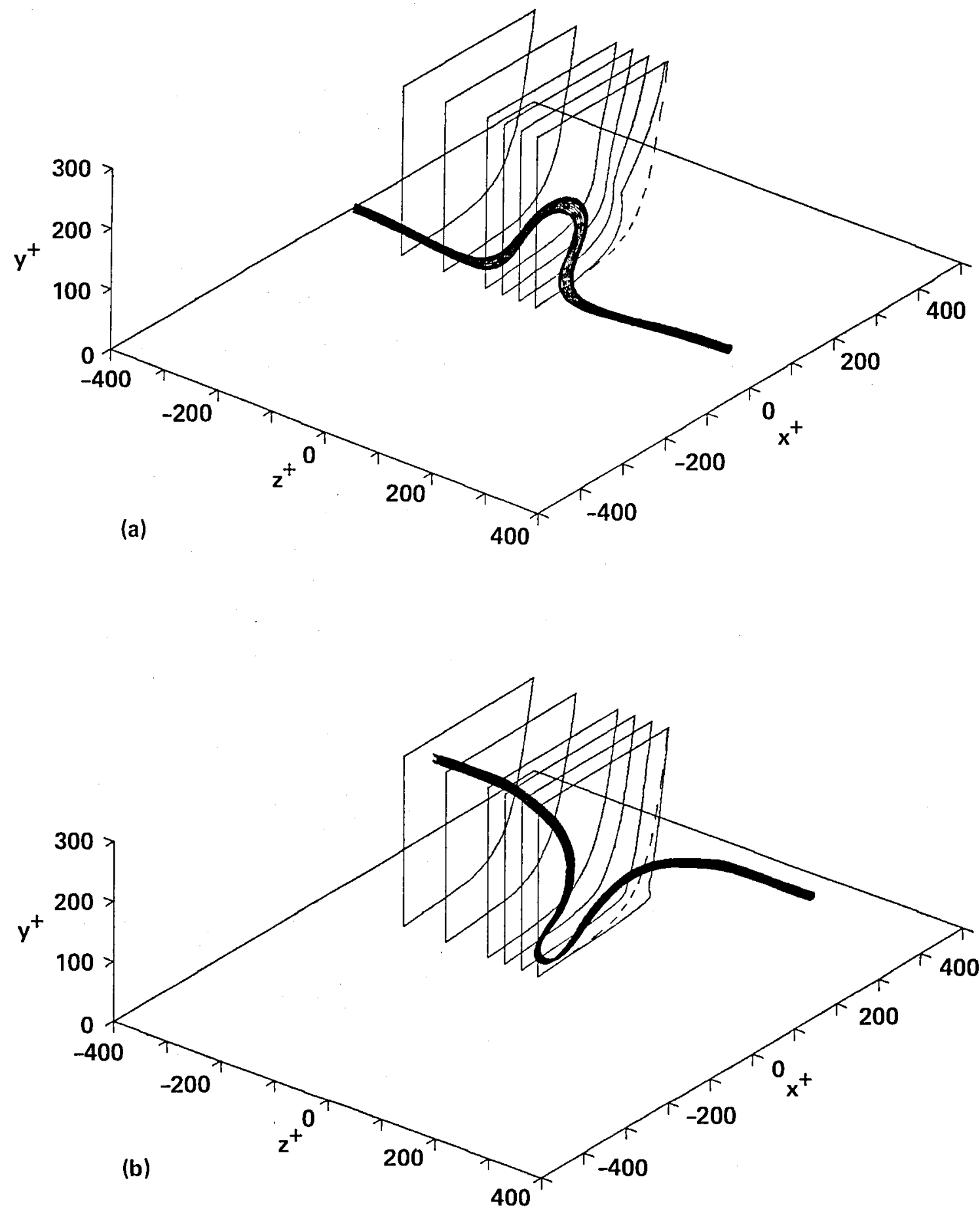

Figure 15. A three-dimensional display of the ensemble-averaged velocity profiles associated with the horseshoe vortex structure: (a) QD-2; (b) QD-4. 

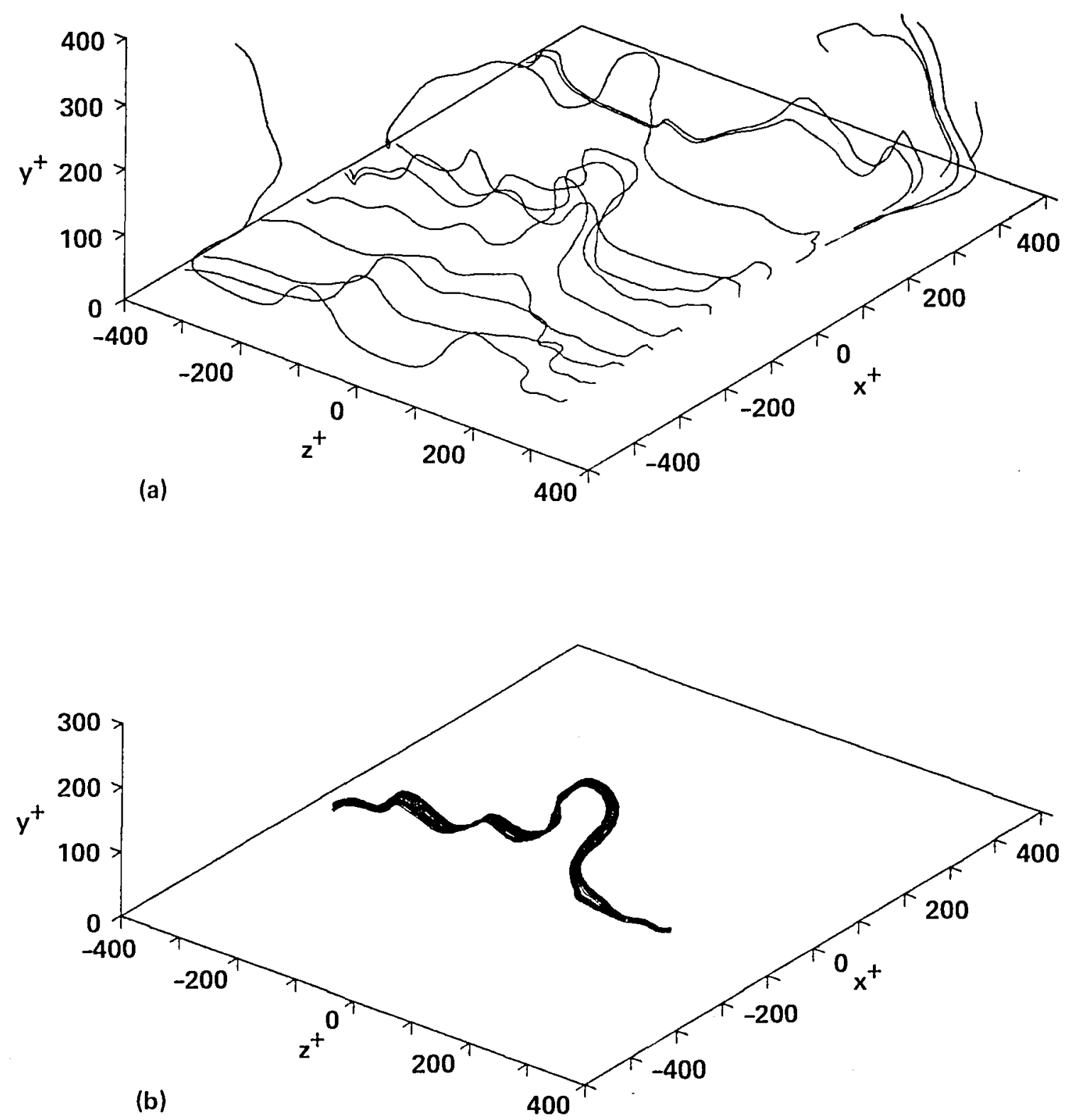

Figure 16. The horseshoe vortex structure detected by QD-2: (a) instantaneous vortex lines; (b) a vortex filament in the vicinity of the detection point. These figures were obtained in the same way as were figures 9 and 10: figures 9 and 10 are from the ensemble-averaged field whereas these are from an instantaneous field. 


\begin{tabular}{|c|c|c|c|}
\hline $\begin{array}{l}\text { 1. Report No. } \\
\text { NASA TM-86657 }\end{array}$ & 2. Government Accession No. & \multicolumn{2}{|c|}{ 3. Recipient's Catalog No. } \\
\hline \multicolumn{2}{|c|}{$\begin{array}{l}\text { 4. Title and Subitle } \\
\text { THE STRUCTURE OF THE VORTICITY FIELD IN TURBULENT } \\
\text { CHANNEL FLOW. PART } 2 \text {. STUDY OF ENSEMBLE-AVERAGED } \\
\text { FIELDS }\end{array}$} & \multicolumn{2}{|c|}{ 6. Performing Organization Code } \\
\hline \multicolumn{2}{|c|}{$\begin{array}{l}\text { 7. Author(s) } \\
\text { John Kim and Parviz Moin }\end{array}$} & \multicolumn{2}{|c|}{$\begin{array}{l}\text { 8. Performing Organization Report No. } \\
85014\end{array}$} \\
\hline \multirow{2}{*}{\multicolumn{2}{|c|}{$\begin{array}{l}\text { 9. Performing Organization Name and Address } \\
\text { NASA Ames Research Center } \\
\text { Moffett Field, CA } 94035\end{array}$}} & \multicolumn{2}{|l|}{ 10. Work Unit No. } \\
\hline & & \multicolumn{2}{|c|}{ 11. Contract or Grant No. } \\
\hline \multirow{2}{*}{\multicolumn{2}{|c|}{$\begin{array}{l}\text { 12. Sponsoring Agency Name and Address } \\
\text { National Aeronautics and Space Administration } \\
\text { Washington, DC } 20546\end{array}$}} & \multicolumn{2}{|c|}{$\begin{array}{l}\text { 13. Type of Report and Period Covered } \\
\text { Technical Memorandum }\end{array}$} \\
\hline & & \multicolumn{2}{|c|}{$\begin{array}{l}\text { 14. Sponsoring Agency Code } \\
505-31-01\end{array}$} \\
\hline \multicolumn{4}{|c|}{$\begin{array}{l}\text { 15. Supplementary Notes } \\
\text { Point of Contact: John Kim, Ames Research Center, MS 202A-1, Moffett Field, } \\
\text { CA } 94035 . \quad \text { (415) } 965-6420 \text { or FTS 448-6420 }\end{array}$} \\
\hline \multicolumn{4}{|c|}{$\begin{array}{l}\text { 16. Abstract } \\
\text { Several conditional sampling techniques are applied to a data base } \\
\text { generated by large-eddy simulation of turbulent channel flow. It is shown } \\
\text { that the bursting process is associated with well-organized horseshoe vor- } \\
\text { tices inclined at about } 45^{\circ} \text { to the wall. These vortical structures are } \\
\text { identified by examining the vortex lines of three-dimensional, ensemble- } \\
\text { averaged vorticity fields. Two distinct horseshoe-shaped vortices corre- } \\
\text { sponding to the sweep and ejection events are detected. These vortices are } \\
\text { associated with high Reynolds shear stress and hence make a significant } \\
\text { contribution to turbulent energy production. The dependency of the ensemble- } \\
\text { averaged vortical structures on the detection criteria, and the question of } \\
\text { whether this ensemble-averaged structure is an artifact of the ensemble- } \\
\text { averaging process are examined. The ensemble-averaged pattern of these } \\
\text { vortical structures that emerge from the analysis could provide the basis } \\
\text { for a hypothetical model of the organized structures of wall-bounded shear } \\
\text { flows. }\end{array}$} \\
\hline \multicolumn{2}{|c|}{$\begin{array}{l}\text { 17. Key Words (Suggested by Author(s)) } \\
\text { Turbulence } \\
\text { Organized structures }\end{array}$} & \multicolumn{2}{|c|}{$\begin{array}{l}\text { 18. Distribution Statement } \\
\text { Un1imited } \\
\text { Subject category - } 34\end{array}$} \\
\hline $\begin{array}{l}\text { 19. Security Classif. (of this report) } \\
\text { Unclassif ied }\end{array}$ & $\begin{array}{l}\text { 20. Security Classif. (of this page) } \\
\text { Unclassif ied }\end{array}$ & $\begin{array}{l}\text { 21. No, of Pages } \\
41\end{array}$ & $\begin{array}{l}\text { 22. Price" } \\
\mathrm{A} 03\end{array}$ \\
\hline
\end{tabular}

-For sale by the National Technical Information Service, Springfield, Virginia 22161 
End of Document 